九州大学学術情報リポジトリ

Kyushu University Institutional Repository

\title{
Vorläufige Mitteilung über des Problem der Ootsuji- und Ashiya-Stufe des Alttertiärs von Kyushu
}

Takahashi, Kiyoshi

Faculty of Sciences, Kyushu University

https://doi.org/10.5109/1524333

出版情報：九州大學理學部紀要：Series D, Geology. 9 (3)，pp.165-182，1960-03-25. Faculty of Science, Kyushu University バージョン：

権利関係 : 
Mem. Fac. Sci., Kyushu Univ., Ser. D, Geology, Vol. IX, No. 3, pp. 165-182, text-figs. 1-5, tables 1-4, pls. 16-18, March 1960

\title{
Vorläufige Mitteilung über das Problem der Ootsuji- und Ashiya-Stufe des Alttertiärs von Kyushu*
}

von

\author{
Kiyoshi TAKAHASHI
}

\section{Einleitung}

Seit langer Zeit wurden die alttertiären Schichten von Kyushu von vielen Forschern untersucht. T. NAGAO (1926-28) und H. Matsushita (1949) haben zusammenfassende Untersuchungen gemacht, die man noch heute als wichtige Arbeiten ansehen kann. Vor kurzem wurde erneut an der Erforschung der fossilen Muschelschalen. reste und der Untersuchung der Foraminiferen erfolgreich gearbeitet und viele neue Tatsachen wurden dadurch herausgebracht. Aber es besteht das grosse noch ungelöste Problem, das mit der Ootsuji- und Ashiya-Zeit zusammenhängt; mit anderen Worten kann man es auch als "Problem der Kishi ma-Schichten " bezeichnen. Der Verfasser hat die alttertiären Schichten von Kyushu nach der pollenstratigraphischen Methode erforscht und das Vorhandensein des sehr wichtigen Problems in der Ootsuji- und Ashiya-Stufe festgestellt. Er möchte hier auf dieses Problem hinweisen und zugleich über die Forschungsergebnisse hinsichtlich der fossilen Pollen und Sporen aus den Schichten der Ootsuji- und Ashiya-Stufe berichten.

Bei dieser Arbeit ist der Verfasser den Professoren H. Weyland (Köln), T. Matsumoto, H. Matsushita, R. Toriyama und M. Noda und den Assistent-Professoren T. Yamasaki und T. Shuto zu grossem Dank verpflichtet. Er muss hier besonders vielen Herren folgender Kohlenbergwerke für ihre freundliche Hilfe beim Sammeln der Arbeitsmaterialien von ganzem Herzen danken: Nittan-Takamatsu, Shin-Ootsuji und Sawara Kohlenbergwerk.

\section{Geologische Aufgabe in der Ootsuji- und Ashiya-Stufe}

Der Verfasser gibt hier eine Übersicht von den Untersuchungen des Alttertiärs, besonders der Schichten der Ootsuji- und Ashiya-Stufe von Nordkyushu und berührt den Kern des Problems.

Zuerst hat T. NAGAo (1927) die obere Pecten sakitoensis-Zone in den Kishima-

* Received November 30, 1959

Der Verfasser hat über Cen Umriss dieser Untersuchung, Februar 1958, in der Jahres. tagung der westjapanischen Abteilung der japanischen geologischen Gesellschaft berichtet und diese Abhandlung im geologischen Institut der Universität Köln, Deutschland ge. schrieben. 
Schichten durch die Erforschung der Muschelschalenreste aus den Kishima-Schichten festgestellt, die Athleta japonica-Zone in den Kyuragi-Schichten und auch die untere Pecten sakitoensis-Zone in den fast obersten Kyuragi-Schichten. Er hat viele marine Molluskenfossilien aus jeder Fossilschicht veröffentlicht.

Die Muschelschalenreste aus den Kishima-Schichten bestehen aus vielen Spezies der alten Typen, die man in der unteren Pecten sakitoensis- und Athleta japonica-Zone finden kann. Mann kann diese Spezies in höheren Schichten als Kishima nicht finden, sondern nur bis zu den Kishima-Schichten.

Anderseits treten wenige Spezies erst in diesen Schichten auf und weiter nach oben; d. h. die Kishima-Schichten zeigen die Zeit an, in der alte Spezies verschwinden und neue Arten auftreten. Trotz der genannten Beschreibung der. Fossilien der Kishima-Schichten, bedeutet es für den Vergleich dieser Ablagerungen mit der Ashiya-Schichtengruppe im Chikuho-Kohlenfeld eine Sicherung, dass T. Nagao die Ablagerungen von den Kishima-Schichten bis den Hatazu-Schiefertonen im KaratsuKohlenfeld "Ashiya-Schichtengruppe “ genannt hat.

H. Matsushita (1949) hat der Ashiya-Schichtengruppe Nagaos im KaratsuKohlenfeld den Namen „Kishima-Schichtengruppe“ gegeben und diese Schichtengruppe noch weiter geteilt, aber er hat die Kishima-Schichtengruppe mit der Ashiya-Schichtengruppe im Chikuho-Bezirk verglichen. Er hat auch einige der Kohlenlager führenden Schichten in die obere Ootsuji-Stufe eingeordnet. Durch diesen Vergleich hat er die grosse gleichzeitige Regression in einigen Kohlenfeldern von Kyushu betont.

A. Mizuno (1956) hat die alttertiären Schichten von Nordwestkyushu durch die erneute Untersuchung der fossilen Muschelschalenreste in drei Zonen, d. h. Venericardia nipponica-Zone (V-n Zone), Venericardia yoshidai-Zone (V-y Zone) und Venericardia vestitoides-Zone ( $\mathrm{V}-\mathrm{v}$ Zone), geteilt. Hier bezieht sich die zweite und dritte Zone auf die Aufgabe des Verfassers. Im Karatsu-Kohlenfeld hat er die. KishimaSchichten und die Oochi-Schichtengruppe im ganzen in die Venericardia yoshidaiZone gestellt und die Schichten vom Sari-Sandstein bis zum Hatazu-Schieferton mit der Venericardia vestitoides-Zone zusammengefasst. Dieses Ergebnis ist sehr interessant, aber es ist sehr bedauerlich, dass er über die Verbindung der Schichten in einigen Kohlenfeldern von Nordostkyushu gar nichts geschrieben hat. Seine Untersuchung darüber wird in kurzem veröffentlicht, nachdem er in den letzten Jahren viele Fossilien in nördlichem Bezirk von Chikuho gesammelt hat.

Der Verfasser ist davon überzeugt, dass die Muschelschalenreste aus den Yamaga-Schichten mit denen der Kishima-Schichten nicht identisch sind und un$z$ weifelhaft $z u$ dem Ashiya-Typus gehören. In den letzten Jahren hat er aus den die Kohlenlager führenden Schichten* unter der Ashiya-Schichtengruppe und einigen anderen gleichalterigen Schichten* ziemlich viele Muschelschalenreste des

\footnotetext{
* Onga-Schichten im Chikuho-Kohlenfeld und Atago-Schichten im Fukuoka-Kohlenfeld.
} 
Ashiya-Typus gesammelt.

Anderseits wurden nach der Untersuchung der Foraminiferen yon K. Asavo und S. Murata (1956) viele Arten aus den Kishima-Schichten schon in den IoojimaSchichten der Ootsuji-Stufe gefunden; die Kishima-Foraminiferengruppe hat allmählich von den oberen Ioojima-Schichten an zugenommen, d. h. diese Fossiliengruppe ist, wie es von den fossilen Muschelschalen erwartet wurde, in der OotsujiStufe aufgetreten und hat sich in der Kishima-Stufe am meisten vermehrt. Die Autoren sind auch zum Schluss gelangt, dass die Kishima-Schichten mit gewisser Möglichkeit zum Aquitan gehören. Weiter haben sie 1957 über die Foraminiferen aus der Ashiya-Schichtengruppe vorläufig berichtet: die Foraminiferen aus dem Orio-Sandstein der untersten Ashiya-Schichtengruppe zeigen ähnliche Formen wie die der Kishima-Schichten und die aus dem auf dem Orio-Sandstein liegenden Norimatsu-Schieferton wie die aus den Sari-Schichten, sie haben die Elemente von Kishima und zugleich die neuen Elemente, die sich nach oben rasch vermehren. Die Autoren haben auch geschrieben, dass dieser Vergleich der Schichten völlig gesichert ist. Diese Folgerung unterstützt schliesslich den Vergleich Nagaos. Die Eigentümlichkeit der vertikalen (zeitlichen) Veränderung der Foraminiferen im Karatsu-Kohlenfeld ist mit der der Veränderung der fossilen Mollusken gleich, aber im Chikuho-Kohlenfeld sind beide Verhältnisse ganz andere.

Wenn der Verfasser den Hauptpunkt dieser Erörterung durch seine palynologisch-stratigraphische Untersuchung prüft, so ergibt sich ein sehr interessantes Resultat. Er kann nämlich die Pollengruppen aus den die Kohlenflöze führenden Schichten, die unter den marinen fossilreichen Schichten, d. h. die Ashiya-Schichtengruppe, im Chikuho- und Kokura-Kohlenfeld liegen, unter demselben Pollenbild, d. h. Onga-Pollenbild, einordnen, aber er kann die aus der Oochi kohlenführenden Schichtengruppe von dem Onga-Pollenbild unterscheiden. Wenn man nach letztem Pollenbild im Chikuho-Kohlenfeld u. a. forscht, kann man dasselbe Pollenbild in den Ideyama-Schichten im Chikuho-Kohlenfeld und in den Shinbaru-Schichten im Kasuya-Kohlenfeld finden. Es sind nämlich die Schichten mit zwei verschiedenen Pollenbildern in der Ootsuji-Stufe Matsushitas unrichtig eingeordnet worden. Der Unterschied dieser beiden Pollenbilder hat seinen Ursprung nicht in der regionalen Verschiedenheit, sondern deutlich in dem vertikalen Verhältnis. Wenn man, wie schon Mizuno geschrieben hat, die Kishima-Schichten von der Oochi-Schichtengruppe altersmässig nicht unterscheiden könnte, müsste man sie zu dem Karatsu-Pollenbild stellen. Und die Schichten über den Sari-Sandsteinschichten könnten mit den Schichten über den Onga-Schichten von Nordostkyushu verglichen werden. Wenn die Kishima-Schichten auch zu der Ashiya-Stufe gehören würden, würden sie das Onga-Pollenbild zeigen. Da der Verfasser die Befunde über die Pollen und Sporen aus den Kishima-Schichten nicht hat, so lässt sich die Vergleichsmöglichkeit der Kishima-Schichten nur nach einigen mittelbaren Angaben vermuten. 
In den letzten Jahren wurden die Konkordanz und Diskordanz zwischen den die Kohlen führenden Schichten und den marinen von einigen Forschern diskutiert. R. SAiro (1957) hat das Vorhandensein einer Diskordanz zwischen den Kishima- und Yoshinotani-Schichten behauptet und er hat die Krustenbewegung von Prä-Kishima (Prä-Aquitan) als wichtig angesehen. Dagegen ist E. Inove (1958) bei der Erklärung der Veränderung der Gesteinfazies in der Kishima-Schichtengruppe über die Kishima-Schichten $z u$ folgendem Schluss gekommen: 1) Die Veränderung der Sedimentszustände von den oberen Yoshinotani-Schichten bis zu den KishimaSchichten tritt allmählich ein. 2) Man kann in der Zeit der Kishima-Ablagerung den Unterschied der Sedimentszustände im Süd und im Nord des Kohlenfeldes erkennen und noch die lokale Veränderung der Zustände feststellen. Nach der Aufhäufung des Hauptteils von Kishima wurde die bisherige unregelmässige Schwankung des Sedimentsbeckens ruhig, die homogene Aufstiegsbewegung fincet im ganzen Sedimentsbecken statt, die oberste Sandsteinschicht ist aufgelagert und, nachdem der Aufstieg des Beckens den Höhepunkt erreicht hat, wird das unterste Konglomerat der Kurokawa-Sandsteinschichten aufgehäuft, aber das Becken ist nicht verlandet. Seine erstere Meinung lehnt die Prä-Kishima-Bewegung Saitos ab. Die Lage der Grenze zwischen den Kishima- und Yoshinotani-Schichten wurde bis heute oftmals diskutiert. Im Kokura-, Chikuho- und Fukuoka-Kohlenfeld ist auch das gleiche Problem vorhanden. H. Matsushita $(1953,1956)$ hat bei der Erwähnung der Diskordanzverhältnisse des Alttertiärs von Nordkyushu das Vorhandensein der Diskordanz zwischen den Atago- und Meinohama-Schichten kehauptet. Dabei handelt es sich allgemein um die Verhältnisse zwischen den marinen und nicht marinen Schichten. Bei diesem Problem gibt es drei verschiedene Ansichten: nämlich, 1) das Vorhandensein einer Diskordanz, 2) der Konkordanz und 3) man erkennt eine m.ehr oder weniger unebene Grenze, was aber nicht als Diskordanz gewertet, sondern durch die Art, wie die nicht marinen Schichten von den marinen Schichten bedeckt werden, erklärt wird. Mán kann hier alś Beispiele die Verhältnisse zwischen den Kishima- und Yoshinotani-Schichten von Karatsu, zwischen den Yamaga- und Onga-Schichten von Chikuho und Kokura und zwischen den Meinohama- und Atago-Schichten von Fukuoka betrachten. Weiter handelt es sich im Karatsu-Kohlenfeld auch um das Verhältnis zwischen den Sari Sandstein- und Kishima-Schichten. Man muss dieses noch weiter untersuchen. Wenn man die Ausdehung und Anordnung der Schichtengruppe im Chikuho-Kohlenfeld ansieht, kann man die Schichten von Ooyake bis Ideyama nur in den südlichen Bezirken erkennen und kann auch bemerken, dass die Onga-Schichten und die AshiyaSchichtengruppe nur in dem nördlichen Bezirk begrenzt vorhanden sind. Und zwar es ist sehr wichtig, dass die Ashiya-Schichtengruppe immer auf den OngaSchichten vorhanden ist. Auf Grund dieser Tatsache kann man irgend eine physikalische Bewegung zwischen den Ideyama- und Onga-Schichten voraussetzen. 
Das ist von grosser Bedeutung. Weiter trifft das in Bezug auf dasselbe Problem im Kokura-Kohlenfeld zu. Es ist selbstverständlich, dass sich die Ashiya-Schichtengruppe im Kokura-Kohlenfeld auf den Onga-Schichten auflagert. R. SArro (1957) hat einige Pflanzenabdrücke aus den Schichten, die von H. Matsushita (1949) Ideyama-Schichten genannt wurde, gesammelt und besteht auf der Möglichkeit des Vergleichs mit der unteren Sasebo-Schichtengruppe aller Schichten von Kokura, weil er diese Abdrücke zur An ia i-Flor a stellt. Der Verfasser hat früher die Pollen und Sporen aus den unteren Schichten untersucht, aber das ganz anderere Pollenbild als Karatsu-Pollenbild betrachtet. Vielleicht ist das Pollenbild noch jünger. Das Verhältnis der Aufschichtung der Onga- und bisherigen IdeyamaSchichten von Kokura kann man unmittelbar im Feld nicht beobachten. Man wird natürlich annehmen können, dass die Onga-Schichten direkt diskordant auf die Basis-schichten folgen.

Im Fukuoka-Bezirk gibt es auch noch eine ungelöste Frage. R. SÁro (1956) und der Verfasser (1957) haben über sie geschrieben: R. SAito hat nämlich Nokonoshima-, Atago- und Meinohama-Schichten als Meinohama-Schichtengruppe zusammengefasst und sie von den Noma- und Uratani-Schichten getrennt. Der Verfasser hat fossile Pollen und Sporen aus den Atago-Schichten untersucht und darauf hingewiesen, dass sich sie von der Pollengruppe aus den Shinbaru-Schichten von Kasuya unterscheiden.

Es ist viel vernünftiger, anzunehmen, dass in Kasuya die Sedimentation in der Shime-Schichtengruppe zu Ende gekommen ist und in Fukuoka in den UrataniSchichten und dass weiter im Nokonoshima-Saitozaki-Bezirk sich die neuen Schichten anderen Systems aufgehäuft haben.

Wenn man in Bezug auf diese Tatsachen berücksichtigt, dass die Schichten der Ashiya-Zeit im Yuyawan (die Bucht von Yuya)-Kottoi-Bezirk diskordant auf den Basisgesteinen liegen, kann man die geologische Frage bezüglich der AshiyaStufe noch deutlicher erfassen. Die Pollengruppe aus den tertiären Schichten von Nishiichi zeigt unteres Onga-Pollenbild. Falls man die tertiären Schichten von Westjapan auf diese Weise ordnet, kann man eine grosse physikalische Bewegung zwischen der Ootsuji- und Ashiya-Stufe erkennen. Aber diese Sache muss noch weiterhin untersucht werden.

\section{Stratigraphie und Pollenbilder}

Der Verfasser bemüht sich hier, das Problem hauptsächlich von der Seite der Pollenanalyse her zu lösen.

A) Chikuho-Kohlenfeld

Die Verbreitung der Ashiya-Schichtengruppe und der Onga-Schichten ist auf den Norden des Chikuho-Kohlenfeldes begrenzt, in den südlichen Bezirken kann 
man nur die Nogata-Schichtengruppe und Ideyama.Schichten feststellen. Diese Tatsache erregt sehr unsere Aufmerksamkeit. Vor der Erläuterung der die Kohlenflöze führenden Onga-Schichten muss der Verfasser hier über die Pollengruppe aus den Ideyama-Schichten eingehend berichten. In seiner Dissertation vom Jahre 1958 hat K. OотA die Pollenanalyse der Kohlenflöze in der östlichen Gegend von Tagawa des Chikuho-Kohlenfeldes durchgeführt. Es gab zwei verschiedene Meinungen über die Einordnung der Schichten in dieser Gegend, in denen man bis heute Fossilien fast nicht finden konnte: Die eine ist die Meinung, dass diese Schichten $z u$ den Ideyama-Schichten gehören, die andere, dass man sie mit den Onga-Schichten vergleichen kann. Der Verfasser hat einiges Material, das K. OотA.

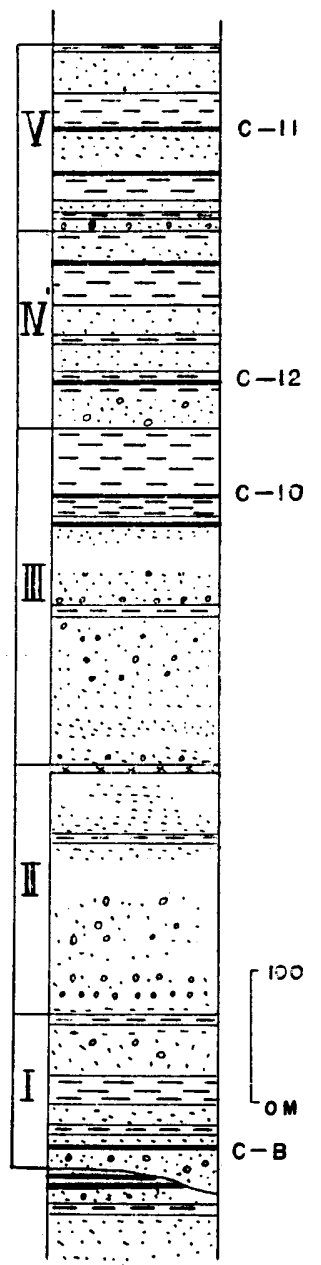

Abb. 1. Profil der Ideyama-Schichten in der östlichen Gegend von Tagawa (K. OotA, 1958) gesammelt und analysiert hat, mit dem Mikroskop untersucht. Er hat dabei eine sehr interessante Tatsache herausgefunden. Im Jahr 1957 hat er vorläufig deren Pollenbestand als "Shin baru$\mathrm{Kish}$ ima-Pollenbild" bezeichnet und im Jahr 1959 diesen Namen in , K a r a t s u-P o 11 e nbi $1 \mathrm{~d}$ " verbessert. Diese Pollengruppe ist mit der des Karatsu-Pollenbildes ganz identisch. Ihr Pollendiagramm ist in der Abbildung 2 dargestellt. Den Charakter des Karatsu-Pollenbildes hat er bereits in seiner anderen Abhandlung eingehend erörtert. Das auffallendeste Merkmal ist die merkwürdige Zunahme der Häufigkeit der Dreieck- und Vieleckpollen. Vor allem tritt Polyvestibulopoll. eminens $\mathrm{T}_{\mathrm{AK}}$. in grösserem Prozentsatz als in anderen Pollenbildern auf und als neue Art tritt Triatriopoll. mirabilis $\mathrm{T}_{\mathrm{AK}}$. in dieser Gruppe auf.

Der Verfasser hat weiter Proben der Yamaura-Kohlen in der Shin-Ootsuji-Grube gesammelt und pollenanalytisch untersucht. In der ShinOotsuji-Grube ist dieses Yamaura-Kohlenflöz sehr dünn und durch die Faltung und den Gleitungsvorgang zertrümmert. Daher konnte er in 10 Präparaten nur 16 Pollen finden. 6 davon sind Polyvestibulopoll. eminens $\mathrm{T}_{\mathrm{AK}}$. Nach dieser Tatsache wird man davon überzeugt sein können, dass dieses Pollenspektrum, obwohl es unvollständig ist, mit grösserer Wahrscheinlichkeit zum Karatsu-Pollenbild gehört. 


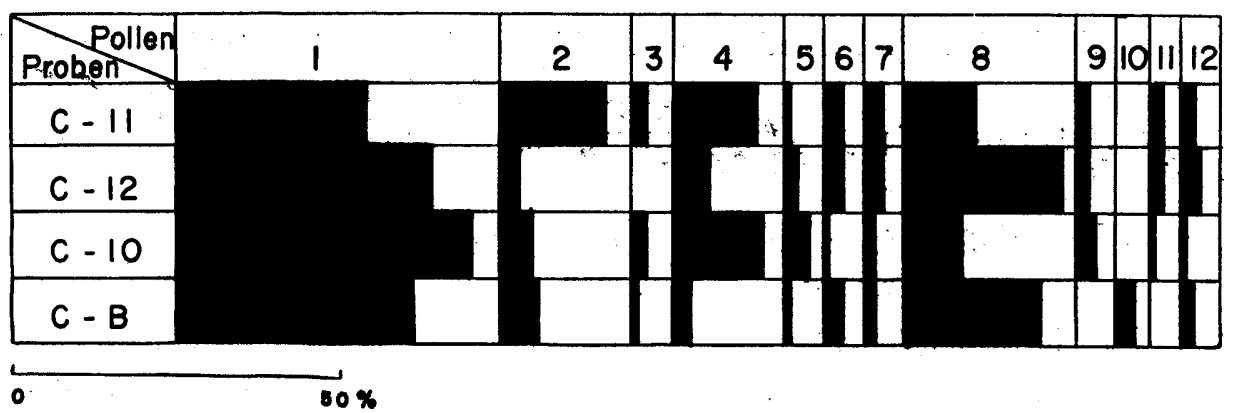

Abb. 2. Pollendiagramm der Ideyama-Schichten in der östlichen Gegend von Tagawa.

1: Inaperturopoll. pseudodubius ТАК.

2: Triporopoll.-Gruppe ( $T$. shimensis, $T$. constatus, $T$. festatus u. a.)

3: Subtriporopoll. kyushuensis TAK.

4: Polyvestibulopoll. eminens TAK.

5: Polyporopoll.-Gruppe ( $P$. grandis und P. undulosus)
6: Tricolpopoll. umiensis ТАK.

7: Tricolpopoll. liblarensis fallax (R. P.oT.)

8: Tricolpopoll. ditis TAK.

9: Tricolpopoll. vulgaris TAK.

10: Tricolporopoll. tertiarius TAK.

11: Triatriopoll. mirabilis TAK.

12: Monocolpopoll.-Gruppe

Tabelle 1. Pollenzähltabelle der Ideyama-Schichten in der östlichen Gegend von Tagawa.

\begin{tabular}{|c|c|c|c|c|}
\hline $\begin{array}{ll}\text { Pollen } & \text { Proben } \\
\end{array}$ & $\mathrm{C}-11$ & C-12 & $\mathrm{C}-10$ & C-B \\
\hline S:ereisporites sp. & 2 & & & 1 \\
\hline Punctatispor. sp. & & & & 1 \\
\hline Reticulatispor sp. & 1 & & & \\
\hline Laevigatospor. dehiscens TAK. & 1 & + & & + \\
\hline Monocolpopoll. universalis TAK. & 2 & 2 & & 2 \\
\hline Monocolpopoll. kyushuensis TAK. & & 1 & 1 & \\
\hline Inaperturopoll. pseudodubius ТАК. & 29 & 39 & 45 & 36 \\
\hline Inaperturopoll. laevigatus TAK. & 2 & 1 & 1 & 1 \\
\hline Pityospor. orientalis TAK. & & 2 & 3 & \\
\hline Triatriopoll. mirabilis TAK. & 2 & 2 & 1 & \\
\hline Triporopoll. constatus ТАK. & 6 & 1 & 2 & + \\
\hline Triporopoll. festatus TAK. & 2 & 1 & 1 & \\
\hline Triporopoll. shimensis TAK. & 7 & 1 & 2 & 3 \\
\hline Triporopoll. sp. & 1 & & & \\
\hline Trsporopoll. sp. & + & & 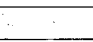 & 3 \\
\hline Subtriporopoll. kyushuensis TAK. & 2 & & 2 & 1 \\
\hline Polyvestibulopoll. eminens TAK. & 13 & 6 & 14 & 3 \\
\hline Polyporopoll. grandis TAK. & 1 & & 2 & \\
\hline Polyporopoll. undulosus (WOLFF) & & 2 & 2 & 1 \\
\hline Tricolpopoll. umiensis TAK. & 3 & 3 & 1 & 3 \\
\hline Tricolpopoll. liblarensis fallax (R. PoT.) & 3 & 3 & 2 & 2 \\
\hline Tricolpopoll. vulgaris ТАK. & 2 & 2 & 3 & \\
\hline Tricolpopoll. ditis TAK. & 11 & 24 & 9 & 21 \\
\hline Tricolpopoll. sculptus TAK. & $1(?)$ & & & \\
\hline Tricolpopoll. reticulatus TAK. & 3 & 1 & 3 & 1 \\
\hline Iricolpopoll. microreticulatus TAK. & 1 & 4 & 2 & 1 \\
\hline Tricolpopoll. rudis TAK. & & & 2 & 2 \\
\hline Tricolpopoll. meinohamensis rotundus TAK. & & 2 & 2 & \\
\hline Tricolpopoll. inamoenus TAK. & & & & 1 \\
\hline Tricolporopoll. cingulum oviformis (R. POT.) & 1 & & & \\
\hline Tricolporopoll. cf. cingulum pusillus (R. POT.) & 1 & & & \\
\hline Taicolporopoll. megaexactus exactus (R. Por.) & & 1 & & \\
\hline Tricolporopoll. tertiarius TAK: & & & & 3 \\
\hline Sonstige & 3 & 2 & 0 & 14 \\
\hline
\end{tabular}

+: Diese Bezeichnung zeigt das Vorhandensein des Pollen, der bei der Zählung nicht aufgetreten ist. 
Das Profil der kohlenführenden Onga-Schichten in Katsuki zeigt die Abbildung 3. Der Verfasser hat 11 Proben der verschiedenen Kohlenflöze der Onga-Schichten in der Shin-Ootsuji-Grube des Ootsuji-Kohlenbergwerkes gesammelt. Weiter im nördlichen Kohlenfeld hat er 10 Proben der Onga-Schichten in der dritten und Tateko (Schacht) Grube des Nittan-TakamatsuKohlenbergwerkes gesammelt. Die Pollenzähltabelle und -diagramm aus diesen Proben lassen folgende Schlussfolgerung $\mathrm{zu}$ : Das Pollenspektrum aus den Onga-Schichten ist mit der Pollengruppe aus den Atago-Schichten von Fukuoka und der OngaSchichten von Kokura, über die er 1957 schon veröffentlicht hat, identisch. Über dieses Pollenspektrum hat er 1957 und 1959 eingehend berichtet und dem Spektrum den Namen „OngaPollen bild " gegeben.

H. Matsushita hat das TakaeKohlenflöz als die Grenze zwischen den Onga- und IdeyamaSchichten festgesetzt, aber der Verfasser möchte das etwas auffallende Konglomerat, etwa $10 \mathrm{~m}$ unter dem Takae-Kohlenflöz, als die Grenze annehmen. Das Pollenspektrum aus der Takae-Kohle zeigt deutlich das Onga-Pollenbild.

a) Onga-Schichten im nördlichen Bezirk

Man kanæ die Onga-Schichten in drei Gruppen einteilen: Ober-, Mittel- und Unterteil. Im Norden

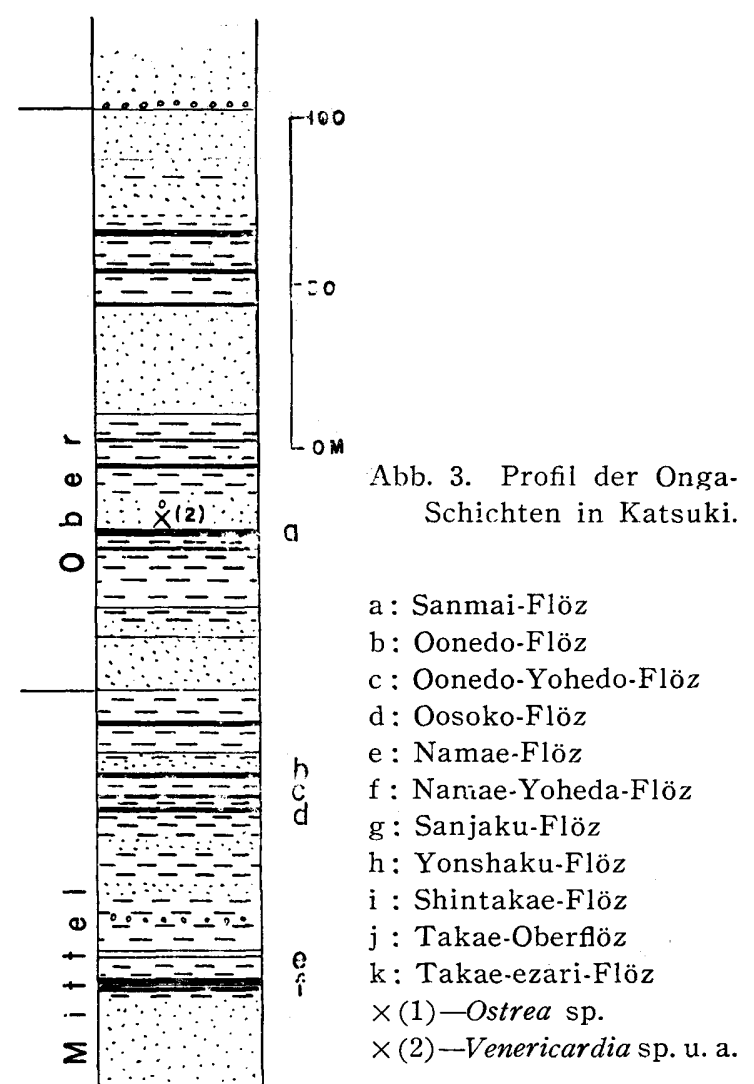

$(1)$ 0

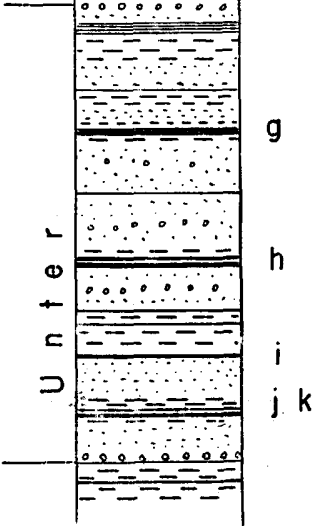
entwickeln sich die dickeren und häufigeren Kohlenflöze besonders in den oberen Schichten, im Süden dagegen werden sie besonders in den mittleren und unteren 


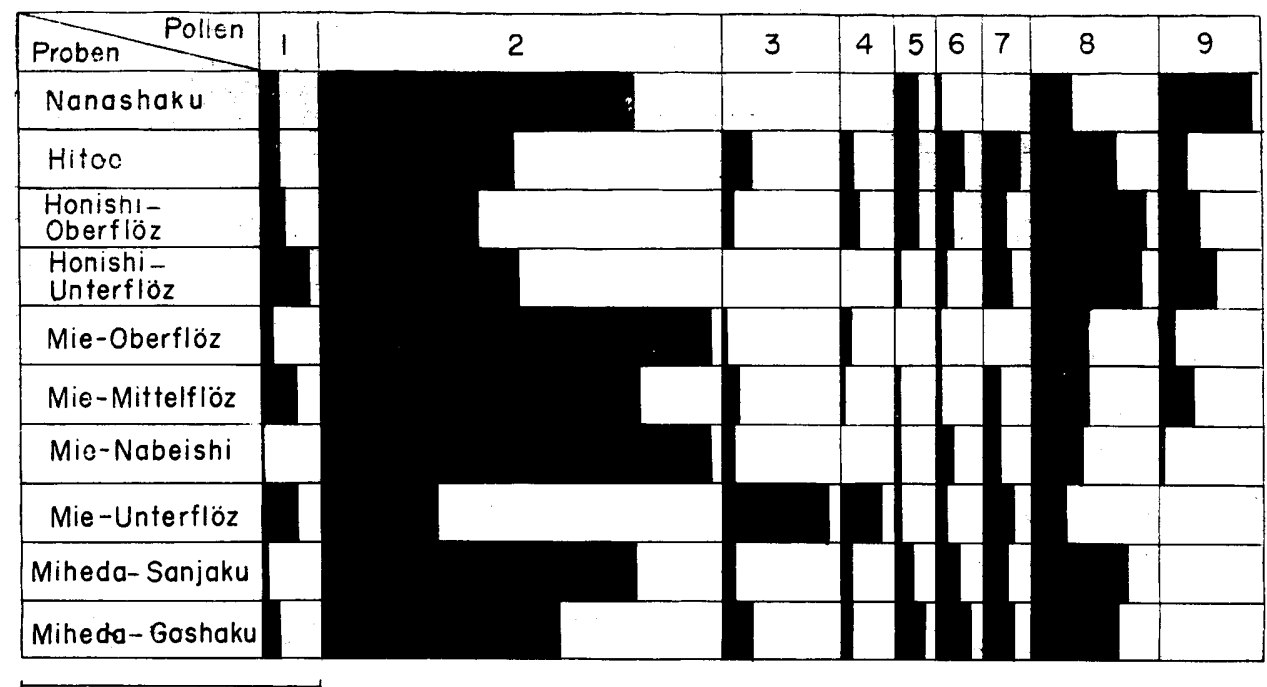

$50 \%$

Abb. 4. Pollendiagramm der Onga-Schichten im nördlichen Bezirk des Chikuho-Kohlenfeldes.

1: Monocolpopoll.-Gruppe

2: Inaperturopoll. pseudodubius TAK.

3: Triporopoll.-Gruppe

4: Polyvestibulopoll. eminens TAK.

5: Tricolpopoll. umiensis $\mathrm{TAK}$.
6: Tricolpopoll. liblarensis fallax (R. PoT.)

7: Tricolpopoll. vulgaris TAK.

8: Tricolpopoll. ditis TAK.

9: Tricolpopoll. meinohamensis meinohamensis TAK.

Schichten dick.

Der Verfasser hat 10 Proben aus der dritten und Tateko (Schacht) Grube des Nittan-Takamatsu-Kohlenbergwerkes gesammelt und untersucht. In deren Pollenspektrum ist der ungeflügelte Koniferen-Pollen Inaperturopoll.pseudodubius TAK. (cf. Taxodiaceae, Cupressaceae u. a.) herrschend. Er reicht hier im Durchschnitt nahe an $45 \%$ heran. Der Pollen vom Inaperturopoll. pseudodubius-Typus wird wahrscheinlich zu dem Kieselholzrest Taxodioxylon sequoianum Gothan in enger Beziehung stehen. Es ist ebenso wie bei Atago von Fukuoka und Onga von Kokura wichtig, dass die Koniferen-Pollen Inaperturopoll. crassatus $\mathrm{T}_{\mathrm{AK}}$. und Inaperturopoll. ligularis TAK. auch hier auftreten.

Die wichtige windblütige Gruppe des extrapalustren Hochwaldes, Tricolpopoll.Typus, steht neben dem Pollen Inaperturopollenites mit einer hohen Frequenz des Auftretens. Der herrschende Pollen der Tricolpopoll.-Gruppe ist Tricolpopoll. ditis TAK. Besonders ist es wichtig, dass der nur in dieser Altersstufe bemerkenswertige Pollen, Tricolpopoll meinohamensis meinohamensis $\mathrm{T}_{\mathrm{AK}}$., in den Onga-Schichten gefunden werden kann. Man kann hauptsächlich im Oberteil der Schichten denselben häufig finden.

Die Dreieck- und Vieleckpollen, die in das Stemma Postnormapolles gruppiert werden, treten mit geringeren Prozentsätzen als bei dem Karatsu-Pollenspektrum 
Tabelle 2. Pollenzähltabelle der Onga-Schichten im nördlichen Bezirk des Chikuho-Kohlenfeldes.

\begin{tabular}{|c|c|c|c|c|c|c|c|c|c|c|}
\hline Proben & A & B & C & $\mathrm{D}^{*}$ & $\mathrm{E}$ & $\mathrm{F}$ & G & $\mathbf{H}$ & I & $\mathrm{J}$ \\
\hline Stereispor. sp. & & & 1 & & & & & & & \\
\hline Laevigatospor. dehiscens TAK. & + & 2 & & & & & & & & 1 \\
\hline Reticuloidospor. (?) sp. & & & & & + & & & & & \\
\hline Monocolpopoll. universalis TAK. & 1 & 1 & 2 & 4 & & 2 & & 2 & & 1 \\
\hline Monocolpopoll. kyushuensis TAK. & 1 & 2 & 1 & 4 & 2 & 4 & & 2 & 1 & 2 \\
\hline Monocolpopoll. intrabaculatus TAK. & 1 & 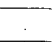 & 1 & & & & & 1 & & \\
\hline Monocolpopoll. sp. & & & & & & & & 1 & & \\
\hline Inaperturopoll. pseudodubius TAK. & 54 & 33 & 27 & 34 & 67 & 55 & 67 & 20 & 54 & 41 \\
\hline Inaperturopoll. laevigatus TAK. & 5 & 2 & 1 & 2 & & 1 & 2 & & & 2 \\
\hline Inaperturopoll. crassatus TAK. & & & $1^{-}$ & 1 & & & & $\overline{1}$ & & \\
\hline Inaperturopoll. ligularis TAK. & & & & & 1 & 1 & 1 & & & \\
\hline Pityospor. orientalis TAK. & 1 & & & + & & & + & + & & \\
\hline Triatriopoll. ongaensis TAK. & & & 2 & & & + & + & 10 & & \\
\hline Triporopoll. shimensis TAK. & & 2 & & & 1 & 2 & 1 & 2 & 1 & 1 \\
\hline Triporopoll. constatus TAK. & & 2 & 2 & & + & 1 & 1 & 5 & & 2 \\
\hline Triporopoll. festatus TAK. & & 1 & & & & & + & 7 & 1 & 2 \\
\hline Triporopoll. ashiyaensis TAK. & & & & & & & & 2 & & \\
\hline Triporopoll. sp. a & & & & & & & & 1 & & \\
\hline Triporopoll. sp. b & & & & & & & & 1 & & \\
\hline Subtriporopoll. kyushuensis TAK. & & & & & & 1 & 1 & 4 & & 1 \\
\hline Subtriporopoll. chikuhoensis TAK. & & & & 2 & & & & & & \\
\hline Polyvestibulopoll. eminens TAK. & & 2 & 3 & & 2 & 1 & + & 7 & 2 & 2 \\
\hline Polyporopoll. grandis TAK. & & & & 1 & & & 1 & & & \\
\hline Polyporopoll. undulosus (WOLFF) & + & & 2 & & $1^{-}$ & & & & & \\
\hline Tricolpopoll. umiensis TAK. & 4 & 4 & 4 & 1 & & 1 & 1 & 1 & 3 & 5 \\
\hline Tricolpopoll. liblarensis (THOMS.) fallax (R. POT.) & 1 & 5 & 3 & 2 & 1 & 1 & 3 & 2 & 4 & 6 \\
\hline Tricolpopoll. vulgaris TAK. & & 6 & 4 & 5 & & 3 & 3 & 5 & 4 & 5 \\
\hline Tricolpopoll. ditis TAK. & 7 & 15 & 20 & 19 & 10 & 10 & 9 & 6 & 17 & 15 \\
\hline Tricolpopoll. reticulatus TAK. & 1 & 1 & 3 & 2 & 2 & 4 & 3 & 3 & 4 & 2 \\
\hline Tricolpopoll. microreticulatus TAK. & & 1 & 8 & & 2 & 1 & 1 & 8 & 3 & 2 \\
\hline Tricolpopoll. rudis TAK. & & & & & & & & 1 & 1 & 4 \\
\hline Tricolpopoll. inamoenus TAK. & $(+)$ & & & & & & & & & \\
\hline Tricolpopoll. meinohamensis meinohamensis TAK. & 16 & 5 & 7 & 10 & 3 & 6 & 1 & & & \\
\hline Tricolpopoll. meinohamensis rotundus TAK. & 2 & & 3 & 1 & & 1 & 1 & & 1 & \\
\hline Tricolpopoll. chikushiensis globulosus TAK. & & & 1 & 1 & & 2 & 1 & & & 1 \\
\hline Tricolpopoll. chikushiensis grandiformis TAK. & 1 & & & + & & & & 1 & & \\
\hline Tricolpopoll. facetus TAK. & & 2 & 1 & & 1 & & & 3 & & 1 \\
\hline $\begin{array}{l}\text { Tricolporopoll. cingulum (R. PoT.) oviformis } \\
\text { (R. Por.) }\end{array}$ & & & & & & & & 1 & & \\
\hline $\begin{array}{r}\text { Tricolporopoll. megaexactus (R. PoT.) exactus } \\
\text { (R. PoT.) }\end{array}$ & & & & & & & & 1 & & \\
\hline Tricolporopoll. inceratus TAK. & 4 & 2 & & & & 1 & & 1 & 1 & \\
\hline Tricolporopoll. asperatus TAK. & 1 & 2 & & & & & & & & \\
\hline Tricolporopoll consularis TAK. & & & 1 & & & & & & & \\
\hline Tricolporopoll. matsushitai TAK. & & & & + & & & & & & \\
\hline Sonstige & 0 & 10 & 2 & 11 & 7 & 2 & 3 & 1 & 3 & 4 \\
\hline
\end{tabular}

+ : Diese Bezeichnung zeigt das Vorhandensein des Pollen, der bei der Zählung nicht aufgetreten ist.

Proben A: Nanashaku-Flöz

B : Hitoe-Flöz

C : Honishi-Oberflöz

D : Honishi-Unterflöz

E : Mie-Oberflöz
F : Mie-Mittelflöz

G: Mie-Nabeishi-Flöz

$\mathrm{H}$ : Mie-Unterflöz

I : Miheda-Sanjaku-Flöz

J : Yoheda-Gosaku-Flöz 
auf. Aber ausnahmsweise kann man im Mie-Unterflöz das Auftreten des Postnormapolles-Typus in verhältnismässig hohem Prozentsatz bemerken. Dieses Merkmal ist in der Karatsu-Pollengruppe sehr ähnlich. Die neue Art, Triatriopoll. ongaensis $\mathrm{T}_{\mathrm{AK}}$, , tritt hier massenhaft auf. Der Tricolporopoll.-Typus ist formenarm. Das ist im Spektrum der Atago- und Kokura-Onga-Scnichten sehr ähnlich. Tricolporopoll. vom Ilex-Typus ist auch selten. Geflügelter Koniferen-Pollen, Pityosporites, tritt nur vereinzelt auf.

Es gilt für das alttertiäre System von Kyushu allgemein, dass die Sporen sehr wenig und formenarm auftreten.

\section{b) Onga-Schichten im südlichen Bezirk}

Im südlichen Bezirk enthält der Unter- und Mittelteil der Onga-Schichten die Hauptkohlenflöze. Daher konnte der Verfasser hauptsächlich Pollen und Sporen aus dem Unter- und Mittelteil der Schichten erforschen. Das ist von grosser Bedeutung, da er sie im Norden aus dem Unter- und Mittelteil der Schichten nicht eingehend untersuchen konnte. Er hat 5 Proben aus dem Unterteil, 5 Proben aus dem Mittelteil und eine Probe aus dem Oberteil pollenanalytisch untersucht.

Glücklicherweise hat er einige Muschelschalenreste von Venericardia in den mittelkörnigen Sandstein des Hangenden des Sanmai-Kohlenflözes sammeln können.

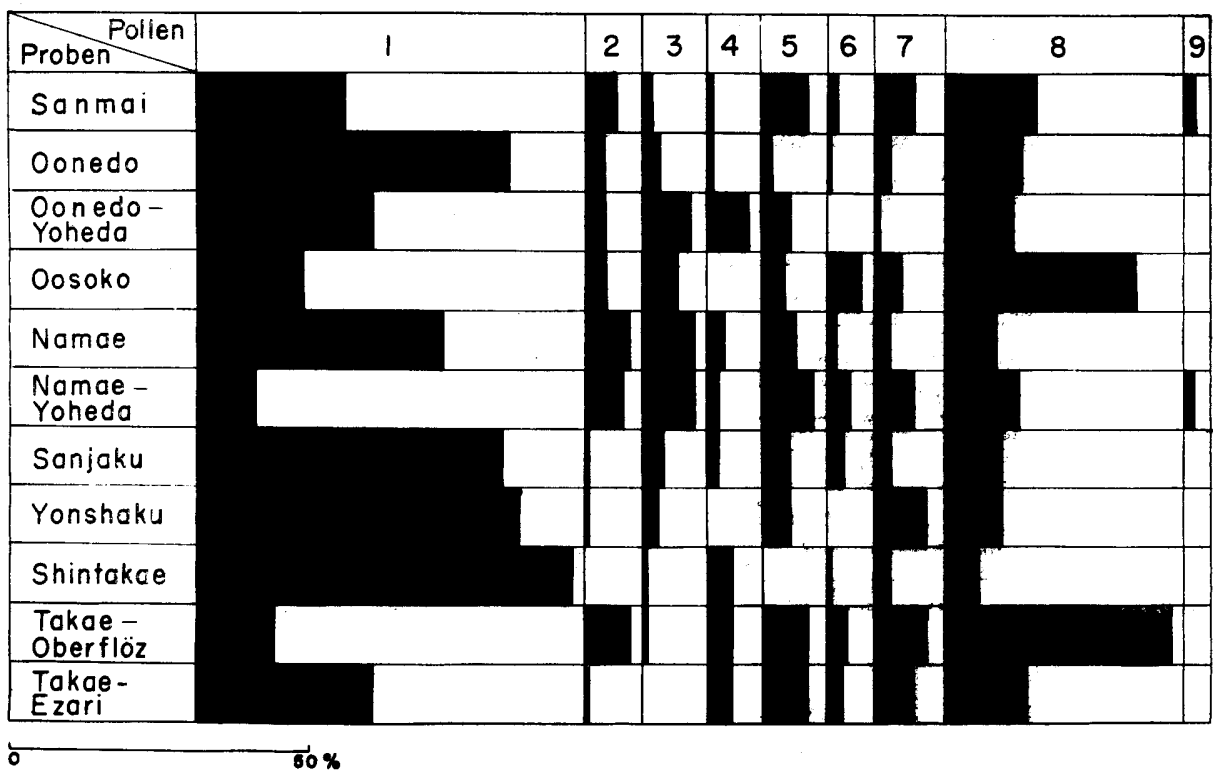

Abb. 5. Pollendiagramm der Onga-Schichten in Katsuki.

1 : Inaperturopoll. pseudodubius ТАK.

2: Monocolpopoll.-Gruppe

3: Triporopoll.-Gruppe

4: Polyvestibulopoll. eminens ТАК.

5: Tricolpopoll. umiensis ТАK.
6: Tricolpopoll. liblarensis fallax (R. РОT.)

7: Tricolpopoll. vulgaris TAK.

8: Tricolpopoll. ditis \TAK.

9: Tricolpopoll. meinohamensis meinohamensis TаK. 
Tabelle 3. Pollenzähitabelle der Onga-Schichten in Katsuki.

\begin{tabular}{|c|c|c|c|c|c|c|c|c|c|c|c|}
\hline Pollen $\quad$ Proben & a & b & c & $\mathrm{d}$ & e & $f$ & g & $\mathrm{h}$ & $\mathbf{i}$ & $\mathrm{j}$ & $\mathrm{k}$ \\
\hline Laevigalospor. dehiscens TAK. & & + & + & & 2 & & & & & & \\
\hline Monocolpopoll. universalis TAK. & 1 & & 1 & 1 & 2 & 2 & & 1 & & 6 & 1 \\
\hline Monocolpopoll. kyushuensis TAK. & 5 & 4 & 3 & 3 & 5 & 2 & 1 & + & & 2 & \\
\hline Monocolpopoll. intrabaculatus TAK. & & & & & 1 & 3 & & & & & \\
\hline Monocolpopoll. sp. & & 1 & & & & 1 & & & & & \\
\hline Inaperturopoll. pseudodubius ТА $\mathrm{K}$. & 25 & 53 & 30 & 18 & 42 & 10 & 52 & $5 \overline{5}$ & 64 & 13 & 30 \\
\hline Inaperturopoll. lqevigatus TAK. & & & 1 & & 1 & & & & $1^{-}$ & & 1 \\
\hline Inaperturopoll. crassatus TAK. & & & & & & & & & $1(?)$ & & \\
\hline Inaperturopoll. ligularis TAK. & & & & & & & 2 & & 3 & & \\
\hline Pityospor. orientalis TAK. & 6 & 2 & 1 & & 2 & & & & & + & \\
\hline Triatriopoll. ongaensis TAK. & & & & & & & & & & 1 & \\
\hline Triporopoll. shimensis TAK. & 1 & & 4 & 2 & 6 & 2 & 2 & 3 & 1 & + & \\
\hline Triporopoll. constatus TAK. & & 2 & 3 & 2 & 2 & 1 & 2 & & + & 1 & \\
\hline Triporopoll. festatus TAK. & 1 & 1 & 1 & 2 & 1 & 6 & & & + & & + \\
\hline Subtriporopoll. kyushuensis TAK. & & & 2 & 1 & & & 3 & 1 & & & \\
\hline Polyvestibulopoll eminens TAK. & 1 & 1 & 7 & & 3 & 2. & 2 & + & 4 & 4 & 4 \\
\hline Polyporopoll. grandis TAK. & & + & 1 & & & + & & & + & & \\
\hline Polyporopoll. undulosus (WOLFF) & . & 1 & + & 1 & 1 & + & 1 & 1 & + & 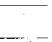 & \\
\hline Tricolpopoll. umiensis TAK. & 8 & 2 & 5 & 4 & 6 & 9 & 5 & 5 & + & 8 & 8 \\
\hline $\begin{array}{l}\text { Tricolpopoll. liblarensis (THOMS.) } \\
\text { fallax (R. РОТ.) }\end{array}$ & 2 & 1 & & 6 & 2 & 4 & 3 & & 1 & 4 & 3 \\
\hline Tricolpopoll. vulgaris TAK. & 7 & 3 & 1. & 5 & 3 & 7 & 3 & 9 & 3 & 9 & 7 \\
\hline Tricolpopoll. ditis TAK. & 16 & 13 & 12 & 33 & 9 & 13 & 10 & 10 & 6 & 39 & 14 \\
\hline Tricolpopoll. reticulatus TAK. & & & 3 & & & 3 & & & 2 & 2 & + \\
\hline Tricolpopoll. microreticulatus ТАK. & 7 & 1 & 4 & 2 & 1 & 2 & 2 & 1 & 1 & 1 & 3 \\
\hline Tricolpopoll. rudis TAK. & & + & + & & 2 & 3 & 2 & 3 & & & 2 \\
\hline Tricolpopoll. inamoenus TAK. & & & & & & & & & & 1 & $1(?)$ \\
\hline Tricolpopoll. meinohamensis meinohamensis & 2 & & & & & 2 & & & & & \\
\hline Tricolpopoll. meinohamensis rotundus TAK. & 4 & 4 & 3 & 6 & 3 & & 1 & 1 & & 1 & 3 \\
\hline Tricolpopoll. chikushiensis globulosus. TAK. & 5 & 1 & 4 & & 1 & 3 & 1 & 3 & 1 & & 2 \\
\hline Tricolpopoll. chikushiensis grandifarmis TAK. & & 1 & & 1 & & 9 & 2 & 3 & & & 2 \\
\hline Tricolpopoll. facetus TAK. & 1 & & & $\bar{\cdot}$ & & 2 & & & & & \\
\hline $\begin{array}{r}\text { Tricolporopoll. cingulum (R. POT.) } \\
\text { oviformis (R. POT.) }\end{array}$ & & & & 3 & 2 & & & & + & 1 & \\
\hline $\begin{array}{c}\text { Tricolporopoll. megaexactus (R. PoT.) } \\
\text { exactus (R. PoT.) }\end{array}$ & & 1 & 2 & 2 & & 1 & & & + & + & \\
\hline Tricolporopoll. inceratus TAK. & & & & & 1 & & & & & & \\
\hline Tricolporopoll. consularis TAK. & & & & & & & & 1 & & & \\
\hline Tricolporopoll. matsushitai TAK. & 2 & 4 & 4 & 5 & 1 & 6 & 1. & + & + & 1 & \\
\hline Tricolporofoll. ongaenșis TAK. & 1 & 1 & & 2 & & 2 & & & & & \\
\hline Tricolporopoll. katsukiensis TAK. & & & & & 1. & & 1 & & 1 & & \\
\hline Sonstige & 5 & 3 & 8 & 1 & 0 . & 5 & 4. & 3 & 11 & 6 & 19 \\
\hline
\end{tabular}

+ : Diese Bezeichnung zeigt das Vorhandensein des Pollen, der bei der Zählung nicht aufgetreten ist.

Proben a : Sanmai-Flöz
b: Oonedo-Flöz
c: Oonedo-yoheda-Flöz
d: Oosoko-Flöz
e: Namae-Flöz
f: Namae-yoheda-Flöz g: Sanjaku-Flöz

h: Yonshaku-Flöz

i : Shintakae-Flöz

j: Takae-Oberflöz

k : Takae-ezari-Flöz 
Diese gehören nicht $z \mathrm{u}$ Venericardia yoshidai $\mathrm{N}_{\mathrm{AGAO}}$, sondern sind nach $\mathrm{T}$. SHuto der Spezies von Venericardia aus der Nichinan-Schichtengruppe sehr ähnlich. Weiter kann man Ostrea-Reste im Unterteil der mittleren Onga-Schichten finden.

Das Pollenspektrum in diesem Bezirk ist dem aus dem Norden sehr ähnlich. Man kann es daher zu demselben Pollenbild stellen.

Tricolpopoll. meinohamensis meinohamensis $\mathrm{T}_{\mathrm{AK}}$. tritt im Sanmai-Kohlenflöz der oberen Onga-Schichten auf, aber auch im Namae-Yoheda-Kohlenflöz der mittleren Onga-Schichten. Im Laufe der Untersuchung kann man immer neue Feststellungen machen und nacheinander neue Spezies bestimmen.

B) Kokura-Kohlenfeld

Der Verfasser hat 1957 Pollen und Sporen aus den Onga-Schichten des KokuraKohlenfeldes veröffentlicht. Das Pollenspektrum aus ihnen kann man als OngaPollenbild auffassen, die Kokura-Onga-Schichten sind nämlich mit den OngaSchichten von Chikuho altersmässig identisch.

R. SAito hat 1957 über einige Pflanzenabdrücke aus den Kokura-IdeyamaSchichten Matsushitas berichtet. Er hat betont, dass diese fossile Flora die Eigentümlichkeiten der Aniai-Flora besitzt und dass deswegen diese Schichten mit dem Unterteil der Sasebo-Schichtengruppe verglichen werden können. Später hat der Verfasser auch einige Pflanzenreste an demselben Ort gesammelt und mit einigen Blattabdrücken aus den Ideyama-Schichten von Chikuho verglichen. Dadurch hat er ermittelt, dass die Flora aus den Kokura-Ideyama-Schichten der aus den Chikuho-Ideyama-Schichten nicht ähnlich und vielmehr jünger ist. $\mathrm{Zu}$ gleicher Zeit hat er auch Pollen und Sporen aus den unteren Ideyama-Schichten von Kokura untersucht. Dieses Pollenspektrum besitzt kein Merkmal des Karatsu-Pollenbildes, dem das Pollenspektrum aus den Ideyama-Schichten von Chikuho angehört. Daher kann er mit Sicherheit sagen, dass Ideyama-Schichten von Kokura mit den Ideyama-Schichten von Chikuho nicht verglichen werden können. Im Feld kann man das wirkliche Verhältnis der Kokura-Ideyama-Schichten zu den Kokura-OngaSchichten nicht beobachten. Man muss darauf achten, dass man in der Grube von Kokura in dem Teil unter dem Hasshaku-Kohlenflöz, das zu dem untersten Kohlenflöz von Kokura gehört, die Rot-Ton-Schichten beobachten kann. Nach der Erfahrung des Verfassers sind in den alttertiären Schichten, die diskordant auf den Basisgesteinen liegen, die rotgefärbten Schichten ausnahmslos mehr oder weniger vorhanden. Auf den Basisschichten in der Ümgebung von Kokura kann man die rotgefärbten Schichten mit ihrer diskordanten Lage bruchstückhaft bemerken, aber beide sind meistens durch Verwerfung gestört. Aus diesem Grund kann man sicher annehmen, dass die Kokura-Onga-Schichten unmittelbar auf den Basisgesteinen liegen. Auf Grund seiner palynologisch-stratigraphischen Untersuchung steht der Verfasser auf einem anderen Standpunkt gegenüber der Meinung, dass 
die Kokura-Ideyama-Schichten Matsushitas mit den Chikuho-Ideyama-Schichten gleichalterig seien.

C) Fukuoka- und Kasuya-Kohlenfeld

Der Verfasser hat schon 1957 Einzelheiten über die Pollen und Sporen aus dem Fukuoka- und Kasuya-Kohlenfeld veröffentlicht und immer geschrieben, dass das Pollenspektrum aus den Shinbaru-Schichten von Kasuya sicher zu dem KaratsuPollenbild gehört.

Im nordwestlichen Fukuoka-Kohlenfeld sind Nokonoshima-, Atago- und Meinohama-Schichten verbreitet, aber Noma- und Uratani-Schichten kommen nur in den südlichen Bezirken vor. Das sagt nicht nur über die geographische Verbreitung etwas aus, sondern hat auch eine erdgeschichtlich wichtige Bedeutung, wenn man auf die stratigraphischen und paläontologischen Untersuchungen eingeht. Im Feld kann man gar nicht feststellen, dass die Atago-Schichten auf den Uratani-Schichten konkordant liegen. Mit anderen Worten: die Sedimentation ist in den UrataniSchichten im Fukuoka-Kohlenfeld und in den Sue-Schichten im Kasuya-Kohlenfeld zum Abschluss gekommen, da man keine konkordanten Schichten auf den Urataniund Sue-Schichten beobachten kann. Der Verfasser hat schon veröffentlicht, dass das Pollenspektrum der Atago-Schichten nicht zu dem Karatsu-Pollenbild gehört, sondern zu dem Onga-Pollenbild. Diese Tatsachen wird man so auffassen können, dass sich im Norden und Süden des Fukuoka-Kohlenfeldes Ablagerungen eines ganz anderen geologischen Systems entwickelt haben. Im Norden kann man ein geologische System, d. h. von den Nokonoshima-Schichten bis zu den MeinohamaSchichten, erfassen. Im Süden gibt es zwei geologische Systeme, nämlich die Fukuoka-Schichtengruppe und die Uratani-Schichten.

Es wurden bereits über viele Muschelschalenreste vom Ashiya-Typus aus den Meinohama-Schichten berichtet, aber der Verfasser konnte neulich aus zwei Horizenten der Atago-Schichten einige Molluskenreste, die vielleicht in die AshiyaFauna eingereiht werden müssen, sammeln. In den Nokonoshima-Schichten hat man ausser einigen Pflanzenabdrücken bis heute kein Fossil gefunden. Wenn man aber die geographische Verbreitung, die Richtung und Neigung der Meinohamaund Atago-Schichten mit denen der Nokonoshima-Schichten vergleicht, kann man sehr leicht verstehen, dass die Nokonoshima-Schichten sehr vernünftigerweise stratigraphisch unmittelbar unter die Atago-Schichten eingesetzt werden.

D) Karatsu-Kohlenfeld

Im Jahre 1959 hat der Verfasser über die Pollen und Sporen aus den OochiSchichtengruppe in Karatsu-Kohlenfeld eingehend berichtet und dabei das Problem der Kishima-Schichten erwähnt. Er hat dieser Pollengruppe den Namen „Ka rat su-Pollenbild " gegeben. Im Chikuho-Kohlenfeld wird dasselbe in den Ide- 
yama-Schichten gefunden. Es unterscheidet sich mit Sicherheit von dem Pollenspektrum der Onga-Schichten im Chikuho- und Kokura-Kohlenfeld und steht gewiss stratigraphisch unter dem Onga-Pollenbild. Da der Verfasser aber Pollen aus den Kishima-Schichten noch nicht nachweisen konnte, so konnte er noch nicht Kritik daran üben, dass die Kishima-Schichten, wie Mızuno (1956) berichtet hat, von der Oochi-Schichtengruppe altersmässig nicht unterschieden werden könnten.

Wenn man geradewegs annähme, dass die Foraminiferengruppe von Kishima mit der aus den Orio-Sandsteinen der Yamaga-Schichten im Chikuho-Kohlenfeld, über welche beiden Gruppen von Foraminiferen Asano und Murata (1956 \& 1957) vorläufig geschrieben haben, verglichen werden kann, müssten die YoshinotaniSchichten von Karatsu und die Onga-Schichten altersmässig gleich sein. Es ist aber erwiesen, dass das Pollenspektrum aus den Yoshinotani-Schichten mit dem aus den Onga-Schichten nicht verglichen werden kann. Murata hat einmal dem Verfasser persönlich gesagt, dass er die Gleichalterigkeit der Foraminiferengruppe aus den Orio-Sandsteinen und der Foraminiferengruppe von Kishima nicht für ganz sicher hält.

Es gibt also jetzt zwei Möglichkeiten für den Vergleich der Schichten in beiden Kohlenfeldern: entweder die Oochi-Schichtengruppe ist mit den IdeyamaSchichten gleichalterig oder die Kishima-Schichten und die Oochi-Schichtengruppe sind mit den Ideyama-Schichten altersmässig gleich.

E) Tertiäre Schichten in einigen sonstigen Gegenden

Über die Pollen und Sporen aus dem Sakito-Matsushima-Kohlenfeld ist die Untersuchung des Verfassers jetzt im Gange. Es ist zu erwarten, dass das Pollenspektrum aus der Matsushima-Schichtengruppe die Eigentümlichkeiten des KaratsuPollenbildes besitzen wird, da durch viele bis heute veröffentlichte Untersuchungen festgestellt worden ist, dass die Schichten im Sakito-Matsushima-Kohlenteld grosse Ähnlichkeit mit denen des Karatsu-Kohlenfeldes haben.

Über die Ashiya-Schichtengruppe von Yuyawan und Kottoi wurde von IMAMURA, WADA u. a. (1951 \& 1952) berichtet. Diese Schichten wurden auf Grund der Muschelschalenreste mit den Ashiya-Schichten von Chikuho verglichen. Man hat schon einige Pflanzenfossilien gefunden, aber pollenanalytische Untersuchungen wurden noch nicht angestellt. Diese alttertiären Schichten sind meistens zusammen mit den Basisgesteinen verworfen, aber es ist klar, dass sich das tertiäre System auf den Basisgesteinen diskordant abgelagert hat.

Aus den nicht marinen tertiären Schichten von Nishiichi ist bis heute kein Fossil bekannt. Es ist nur eine nicht gesicherte Annahme, dass diese Schichten mit den alttertiären Schichten von Ube fast gleichalterig sein könnten. Der Verfasser hat einmal die Gelegenheit gehabt, Kohlenproben, die von MAtsuki gesammelt wurden, pollenanalytisch zu untersuchen. Dabei wurde festgestellt, dass das 
Tabelle 4. Zusammenstellung der Schichten der Ootsuji- und Ashiya-Stufe von Nordkyushu und Westhonshu.

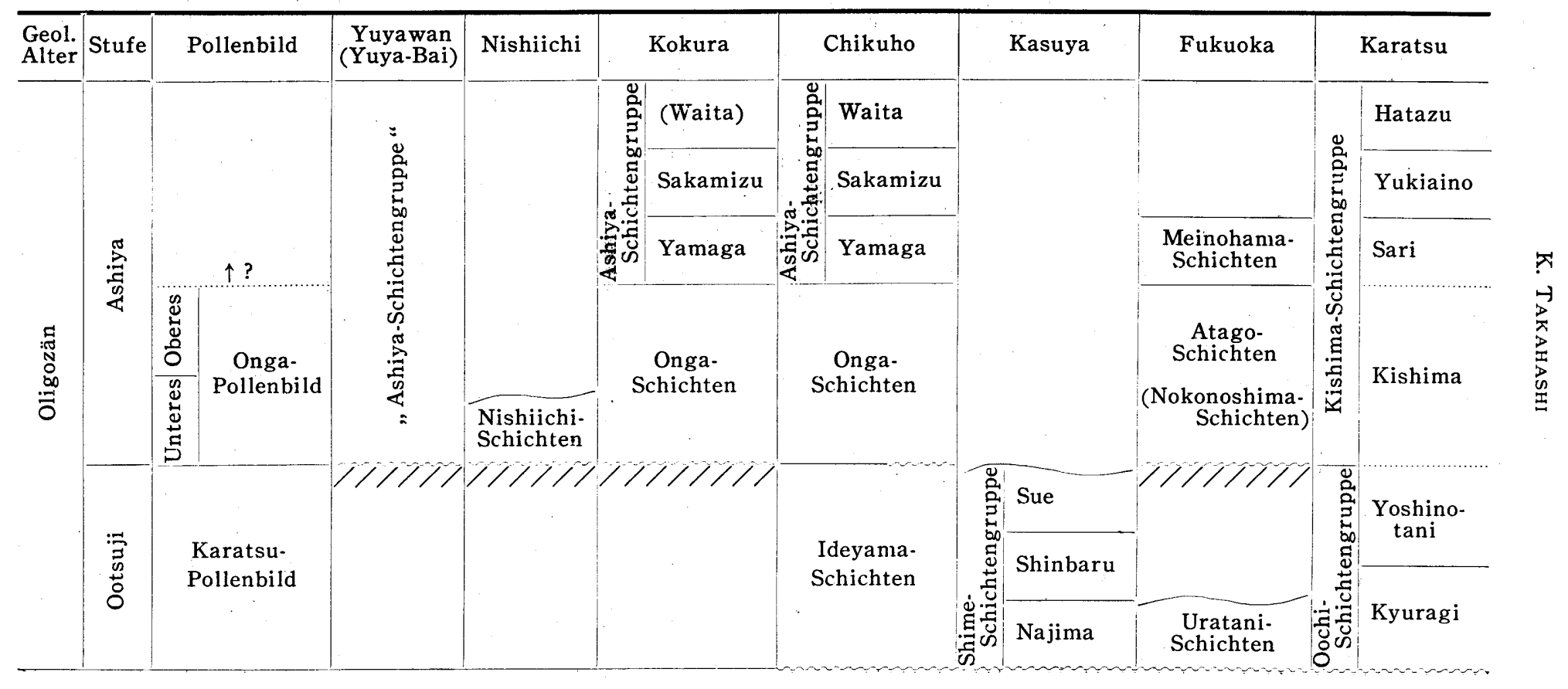


Pollenspektrum das untere Onga-Pollenbild zeigt.

Der Verfasser hat 1958 bei der Beschreibung von Sabalites taishuensis $\mathrm{T}_{\mathrm{AK}}$. über den Umriss der alttertiären Schichten von Tsushima berichtet. S. Kanno hat 1955 geschlossen, dass das geologische Alter der Taishu-Schichtengruppe auf Grund der Muschelschalenreste, die im Inneren einer Grube der Taishu-Mine gefunden wurden, Oligomiozän sei. Er hat über einen Rest von Venericardia yoshidai NAGAO berichtet, dessen Erhaltungszustand nicht sehr gut ist, aber trotzdem wird man darauf hinweisen können, dass die Fauna von Tsushima zu der Fauna vom Ashiya-Typus gehört. Der Verfasser hat noch keinen Erfolg mit der Pollenanalyse der Proben aus der Taishu-Schichtengruppe gehabt.

Wenn man die oben erwähnten Tatsachen zusammenfasst und einen Vergleich der Schichten in den genannten Kohlenfeldern versucht, kann man die folgende Tabelle (Tabelle 4) aufstellen.

\section{Zusammenfassung}

Die Bearbeitung behandelt hauptsächlich das Problem der Ootsuji- und AshiyaStufe des Alttertiärs von Kyushu und ist eine vorläufige Untersuchung, die ausgeführt wurde, um vom Standpunkt der Pollenanalyse aus das Problem zu lösen. Hier wird also hauptsächlich über das Resultat der Pollenanalyse der Schichtengruppe im Chikuho-Kohlenfeld berichtet. Der Vergleich, der durch die Pollenanalyse ermöglicht wurde, wie in der letzten Tabelle gezeigt wurde, wird unser Interesse für die Lösung des Problems der Ootsuji- und Ashiya-Stufe noch vergrössern.

\section{Literatur}

Asano, K. und Murata, S. (1956): Foraminiferen des Alttertiärs im Karatsu-Kohlenfeld (Vorläufige Mitteilung). (jap.), Foraminiferen (Yukoochu) 5, 44-48.

(1957): Die fossilen Foraminiferen aus der Ashiya-Schichtengruppe von Kyushu (Vorläufige Mitteilung). (jap.), Foraminiferen (Yukoochu) 7, 28-31.

InouE, E, (1958): On the Geological Structure and Lithofacies Variation of the Kishima Group in the Karatsu Coal-field. (jap. mit engl. Resumé), Journ. Geol. Soc. Japan, 64, 748, $29-39$.

Kanno, S. (1955): Tertiary Mollusca from Taishu Mine, Tsushima Nagasaki Prefecture, Japan. Trans. Proc. Palueont. Soc. Japan, N.S., 18, 31-36.

Matsushita, H. (1949): Geology of Coal Fields in Northern Kyushu. (jap.), Journ. Mining Kyushu, Spec. edit.

(1952): Der Bezirk des Oonoura und Takamatsu Kohlenbergwerkes im ChikuhoKohlenfeld. (jap.), Führerbuch der geol. Exkursion, geol. Inst. Univ. Kyushu.

(1953): Diskordanz in den Kohlenfeldern von Kyushu. (jap.), Chikyu-Kagaku, 9, 14-21. (1956): Again on the Unconformity in the northern Kyushu Coal-field. (jap. mit eng1. Resumé), Journ. Mining Kyushu, 24, 2, 49-55.

Mizuno, A. (1956): A Preliminary Note on the Megafaunal Zones of the Paleogene in Northwestern Kyushu, Japan (for correlation of the Paleogene formations in Japan). (jap. mit engl. Resumé), Bull. geol. surv. Japan, 7, 6, 25-34. 
Murakoshi, H. (1949): Über die Geologie im Kokura-Kohlenfeld. (jap.), Mineralogie $u$. Geologie, 10, 164-169.

NaGAO, T. (1927-28): Stratigraphie der alttertiären Schichten von Kyushu. (jap.), Journ. Geogr., Nr. 463-468.

Noda, M. and Sujaku, T. (1955): Stratigraphic Relations between the Ashiya, Nishisonogi and Sasebo Groups. (jap. mit engl. Resumé), Journ. Geol. Soc. Japan, 61, 715, 150-161.

OотA, K. (1958): Geology of the Tagawa District, Fukuoka Pref. (jap. mit engl. Resumé), Diss. Kyushu Univ.

Saito, R. (1957): Pre-Kishima (Pre-Aquitanian) Crustal Deformation of Japan and the adjacent circume Pacific regions. Kumamoto Journ. Sci., Ser. B, Sec. 1, Geol., 2, 2, 33-50. - (1957): On the Stratigraphical Horizon of the Coal-bearing Formation of the Kokura Coal-field. (jap. mit engl. Resumé), Journ. Mining Kyushu, 25, 1, 21-22.

TAKAHASH, K. (1957): Palynologisch-stratigraphische Untersuchung der tertiären Schichten im Kasuya und Fukuoka Kohlenfeld von Nordkyushu, Japan. Mem. Fac. Sci. Kyushu Univ., Ser. $D, 5,4,199-221$.

(1957): Pollen und Sporen aus der Onga-Formation im Kokura-Kohlenfeld, Nordkyushu. (jap. mit deutsch. Resumé), Journ. Mining Kyushu, 25, 8, 324-328.

(1957): Pollen und Sporen aus den Hauptflözen im Miike und Asakura Kohlenfeld und ihre stratigraphische Bedeutung. (jap. mit deutsch. Resumé), Journ. Mining Kyushu, 25, $12,508-516$.

- (1958): Sabalites aus den Wakata-Schichten von Tsushima, Nordkyushu. Trans. Proc. Palaeont. Soc. Japan, N.S., Nr. 30, 185-188.

- (1959y: Pollenanalytische Untersuchung der oligozänen Kohlenlager im KaratsuKohlenfeld. (jap. mit deutsch. Resumé), Journ. Mining Kyushu, 27, 11, 474-483.

Thomson, P. W. und Pflug, H. D. (1953): Pollen und Sporen des mitteleuropäischen Tertiärs. Palaeontogr., B., 94, 1-138.

WadA, K., Imamura, S. und Hase, A. (1951): Alttertär in der östlichen Yuya-Bai, Ootsu, Prov. Yamaguchi. (jap.), Journ. Geol. Soc. Japan, 57, 670, 306.

Wada, K. und Imamura, S. (1952): Altertiär im nordwestlichen Bezirk von Toyoura, Prov. Yamaguchi. (jap.), Journ. Geol. Soc. Japan, 58, 682, 307-308.

YAMASAKI, T. (1953): Stratigraphy of the Karatsu Coal Field, Kyushu. (jap. mit engl. Resumé), Journ. Geol. Soc. Japan, 59, 696, 403-479. 
Kiyoshi TAKAHASHI

Vorläufige Mitteilung über das Problem der Ootsuji- und Ashiya-Stufe des Alttertiärs von Kyushu

\section{Tafeln 16-18}




\section{Erklärung zu Tafel 16}

Fig. 1. Stereispor. sp.

Ideyama-Schichten, ca. $810 \times$

Fig. 2-4. Laevigatospor. dehiscens TAK.

Onga-Schichten; Fig. 2, 4: Nanashaku-Flöz, ca. 570x; Fig. 3: Hitoe-Flöz, ca. 810X

Fig. 5. Monocolpopoll. universalis TAK.

Onga-Schichten, Takae-ezari-Flöz, ca. $810 \times$

Fig. 6, 7. Monocolpopoll. kyushuensis TAK.

Onga-Schichten; Fig. 6: Honishi-Unterflöz; Fig. 7: Yonshaku-Flöz; ca. $810 \times$

Fig. 8. Monocolpopoll. intrabaculatus TAK.

Onga-Schichten, Takae-ezari-Flöz, ca. $810 \times$

Fig. 9. Monocolpopoll." sp.

Onga-Schichten, Mie-Unterflöz, ca. $810 x$

Fig. 10-15. Inaperturopoll. pseudodubius TAK.

Onga-Schichten; Fig. 10: Takae-Oberflöz; Fig. 11: Mie-Nabeishi-Flöz; Fig. 12, 14. 15:

Shintakae-Flöz; Fig. 13: Yonshaku-Flöz; ca. 810X

Fig. 16-18. Inaperturopoll. ligularis $\mathrm{TAK}_{\mathrm{A}}$

Onga-Schichten; Fig. 16, 18: Shintakae-Flöz; Fig. 17: Mie-Nabeishi-Flöz; ca. 810X

Fig. 19. Inaperturopoll. laevigatus $\mathrm{TAK}$.

Onga-Schichten, Nanashaku-Flöz, ca. $810 x$

Fig. 20, 21. Pityospor. orientalis TAK.

Onga-Schichten ; Fig. 20: Takae-Oberflöz, ca. 810x ; Fig. 21: Namae-Flöz, ca. 570X

Fig. 22. Triatriopoll. mirabilis TAK.

Ideyama-Schichten, ca. $810 \times$

Fig. 23-27. Triatriopoll. ongaensis TAK.

Onga-Schichten, Mie-Unterflöz, ca. $810 \times$

Fig. 28-30. Triporopoll. shimensis $\mathrm{T}_{\mathrm{AK}}$.

Onga-Schichten, Mie-Unterflöz, ca. $810 x$

Fig. 31, 32. Triporopoll. constatus TAK.

Onga-Schichten ; Fig. 31: Mie-Unterflöz; Fig. 32 : Mie-Oberflöz; ca. 810X

Fig. 33, 34. Triporopoll. festatus TAK.

Onga-Schichten; Fig. 33: Takae-ezari-Flöz; Fig. 34: Namae-Yoheda-Flöz; ca. 810x

Fig. 35. Triporopoll. ashiyaensis $\mathrm{TAK}$.

Onga-Schichten, Mie-Unterflöz, ca. $810 \times$

Fig. 36-39. Polyvestibulopoll. eminens TAK.

Onga-Schichten; Fig. 36: Takae-ezari-Flöz; Fig. 37-39: Shintakae-Flöz; ca. 810x 

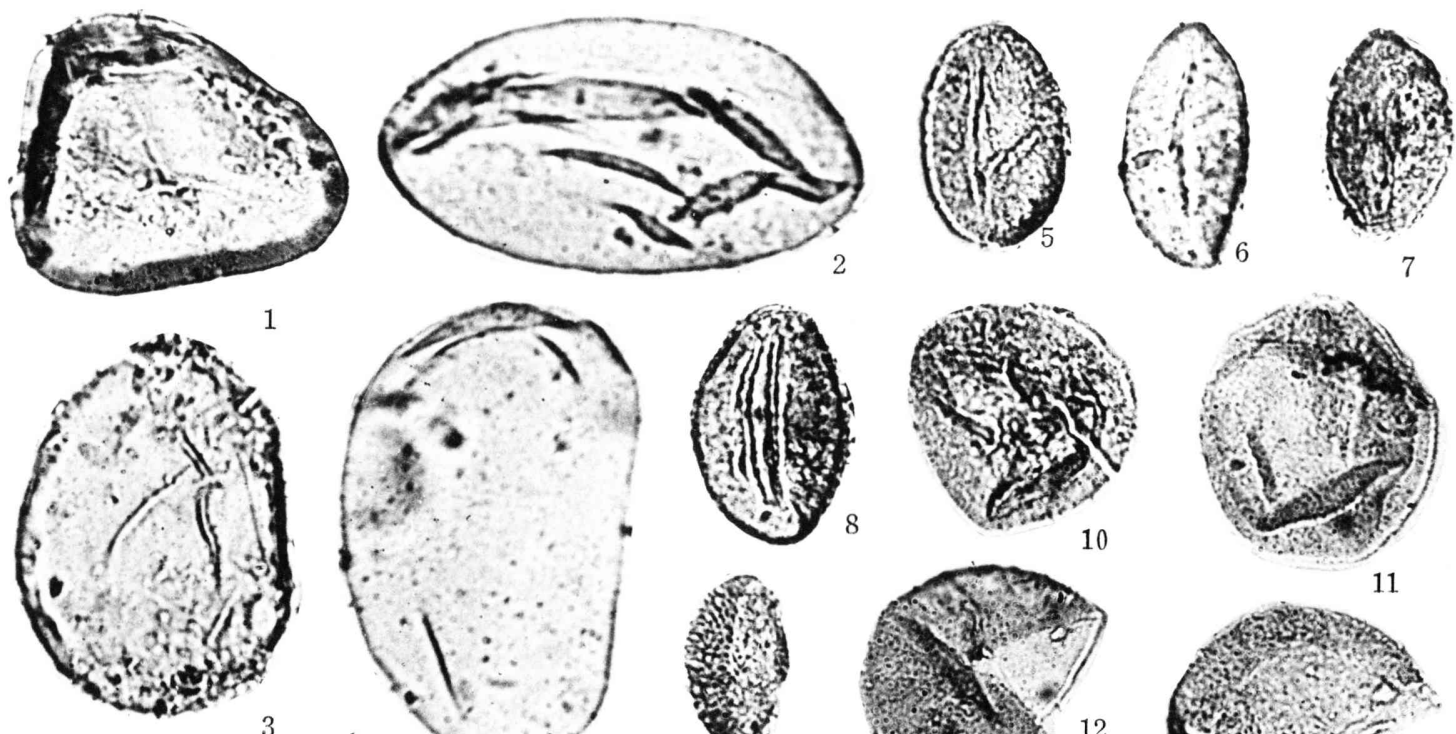

10

11
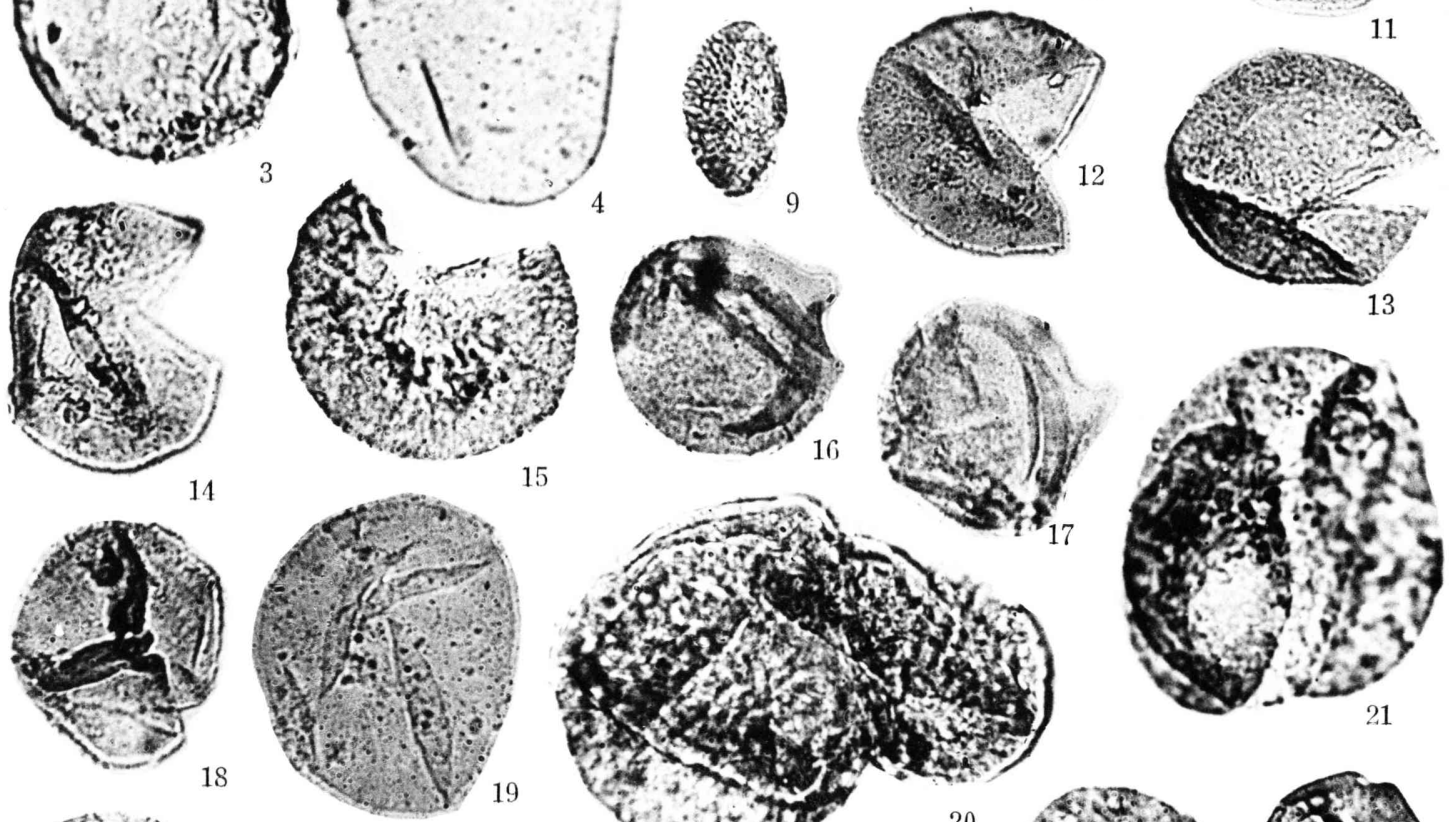

\section{${ }^{15}$}
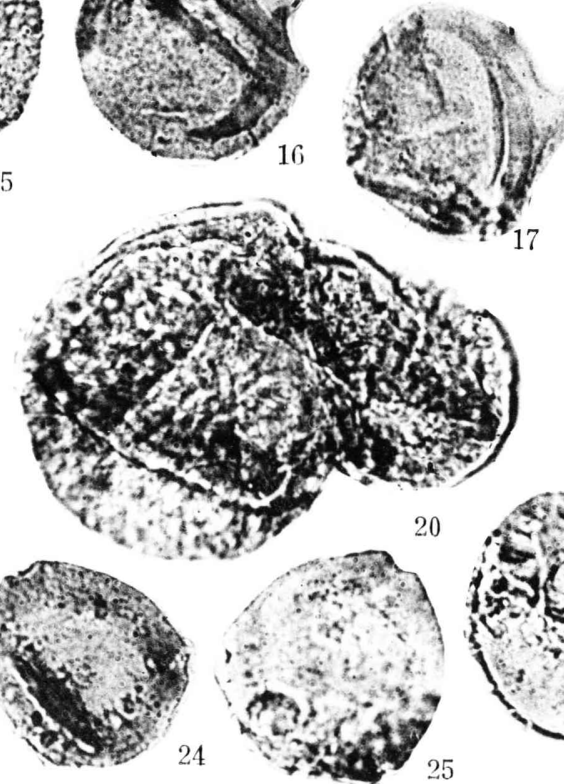

13
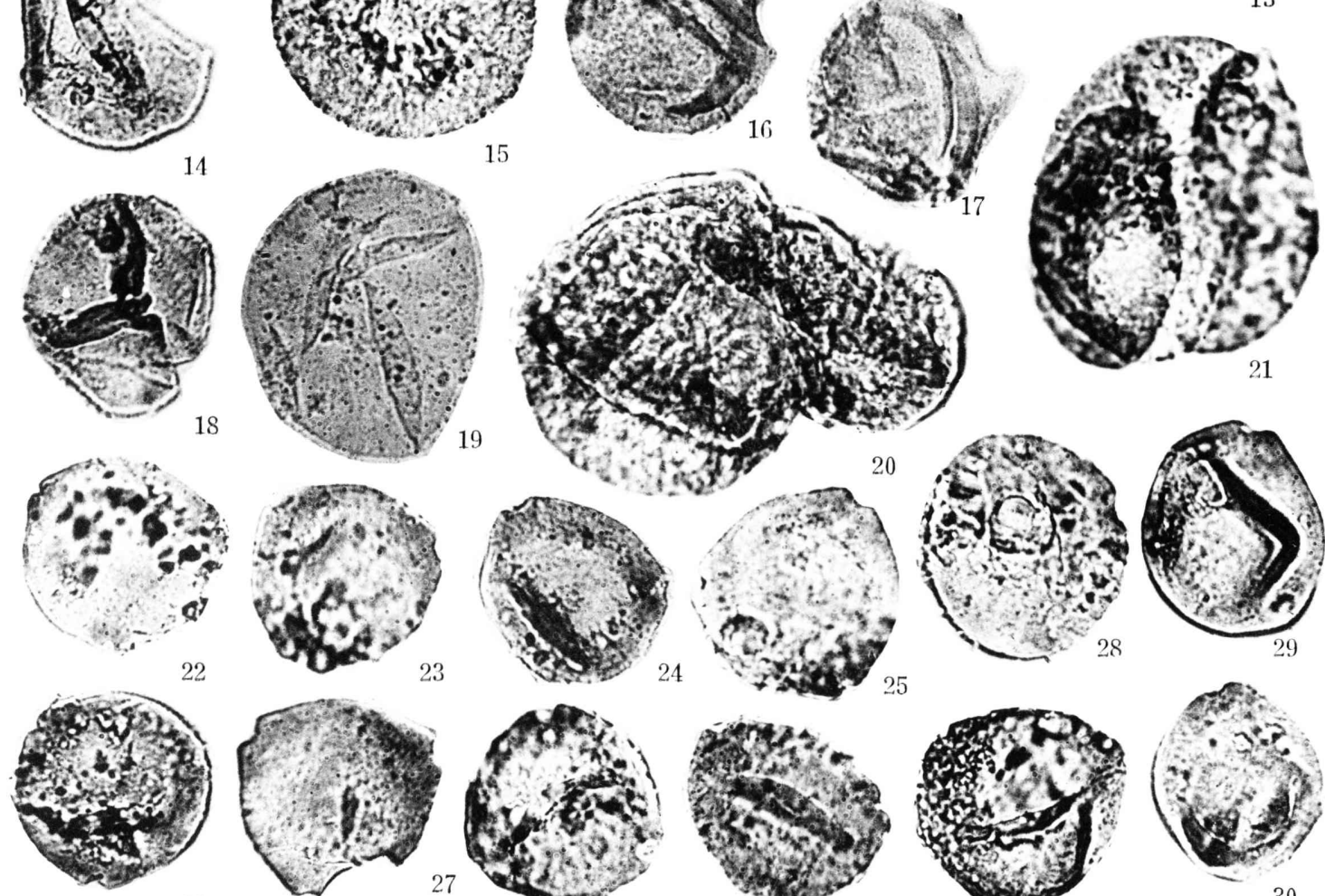

"n
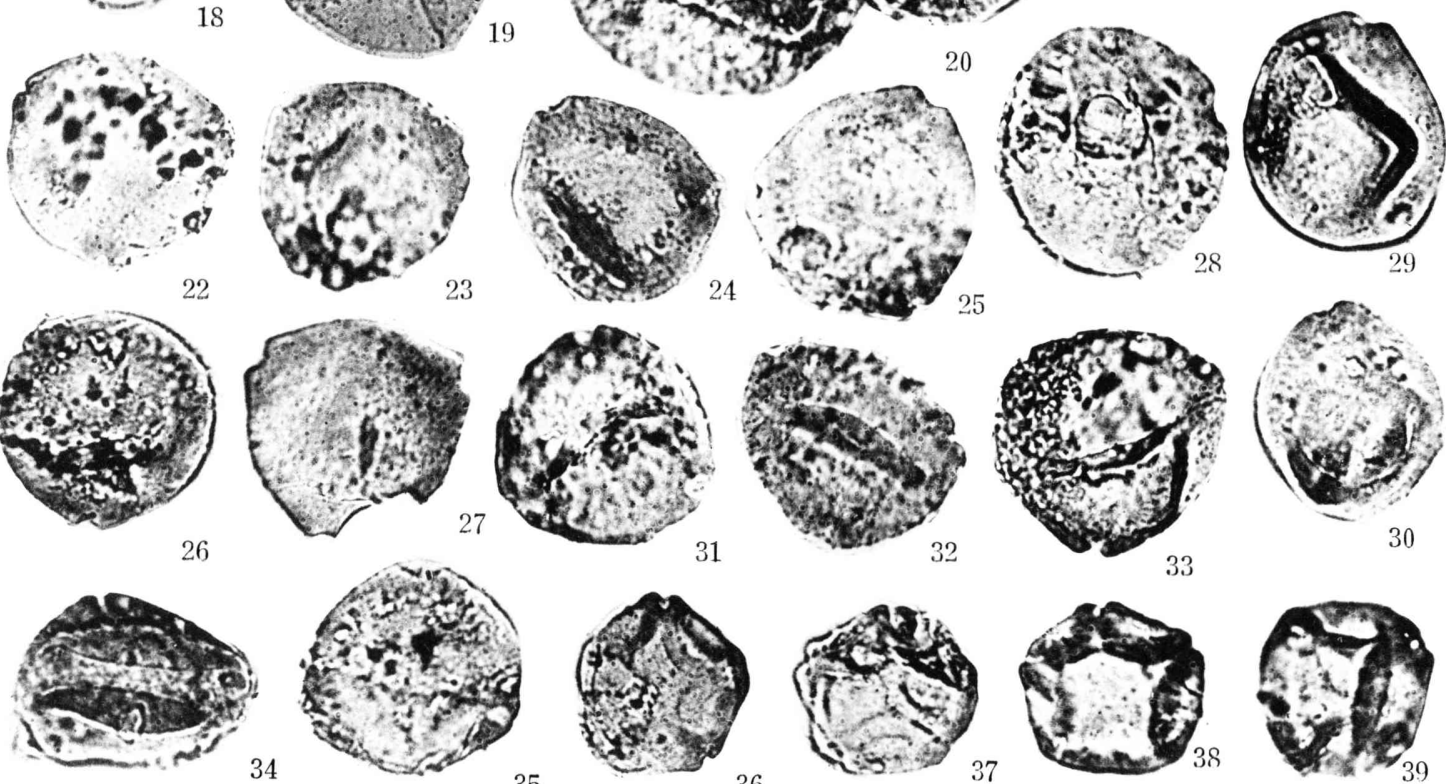

33
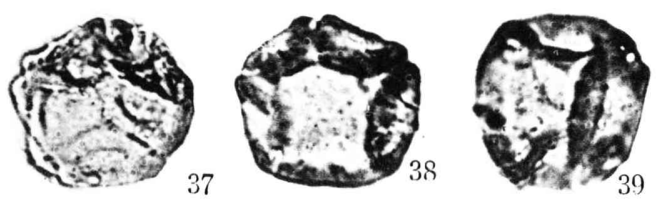

K. Takahashi : Vorläufige Mitteilung über das Problem 


\title{
Erklärung zu Tafel 17
}

\author{
(Alle Objekte ca. $810 x$ )
}

Fig. 1, 2. Polyvestibulopoll. eminens TAK.

Onga-Schichten; Fig. 1: Mie-Unterflöz; Fig. 2: Mie-Nabeishi-Flöz

Fig. 3. Triporopoll. constatus TAK.

Onga-Schichten, Mie-Unterflöz

Fig. 4. Triatriopoll. ongaensis $\mathrm{T}_{\mathrm{AK}}$. Onga-Schichten, Mie-Unterflöz

Fig. 5-8. Polyporopoll. undulosus (WOLFF)

Onga-Schichten, Fig. 5: Honishi-Oberflöz, Fig. 7: Shintakae-Flöz, Fig. 8: Ooneda-YohedaFlöz; Ideyama-Schichten, Fig. $6: \mathrm{C}-\mathrm{B}$

Fig. 9. Subtriporopoll. kyushuensis ТАK. Onga-Schichten, Nanashaku-Flöz

Fig. 10. Polyporopoll. grandis TAK. Onga-Schichten, Honishi-Unterflöz

Fig. 11. Subtriporopoll. chikushiensis TAK. Onga-Schichten, Honishi-Unterflöz

Fig. 12-22. Tricolpopoll. umiensis $\mathrm{T}_{\mathrm{AK}}$. Onga-Schichten, Fig. 12: Oonedo-Yoheda-Flöz, Fig. 13, 20: Shintakae-Flöz, Fig. 14, 22 : Namae-Yoheda-Flöz, Fig. 15: Nanashaku-Flöz, Fig. 16, 18: Takae-ezari-Flöz, Fig. 17: Yonshaku-Flöz, Fig. 19: Sanjaku-Flöz, Fig. 21: Takae-Oberflöz

Fig. 23-29. Tricolpopoll. ditis TAK.

Onga-Schichten, Fig. 23, 24: Takae-ezari-Flöz, Fig. 25-27: Takae-Oberflöz, Fig. 29: MieUnterflöz; Ideyama-Schichten, Fig. 28: C-B

Fig. 30-32. Tricolpopoll. reticulatus TAK.

Onga-Schichten, Fig. 30 : Mie-Unterflöz, Fig. 31: Takae-ezari-Flöz, Fig. 32 : Shintakae-Flöz

Fig. 33-35. Tricolpopoll. microreticulatus Tak.

Onga-Schichten, Takae-ezari-Flöz

Fig. 36-38. Tricolpopoll. liblarensis fallax (R. PотоNIE)

Onga-Schichten. Fig. 36: Namae-Yoheda-Flöz, Fig. 37: Shintakae-Flöz, Fig. 38: TakaeFlöz

Fig. 39, 40. Tricolpopoll. facetus TAK.

Onga-Schichten, Fig. 39: Mie-Unterflöz, Fig. 40: Hitoe-Flöz

Fig. 41. Tricolpopoll. rudis $\mathrm{TAK}_{\mathrm{A}}$.

Onga-Schichten, Takae-ezari-Flöz

Fig. 42, 43. Tricolpopoll. meinohamensis meinohamensis $\mathrm{T}_{\mathrm{AK}}$.

Onga-Schichten, Fig. 42: Nanashaku-Flöz, Fig. 43: Honishi-Unterflöz 

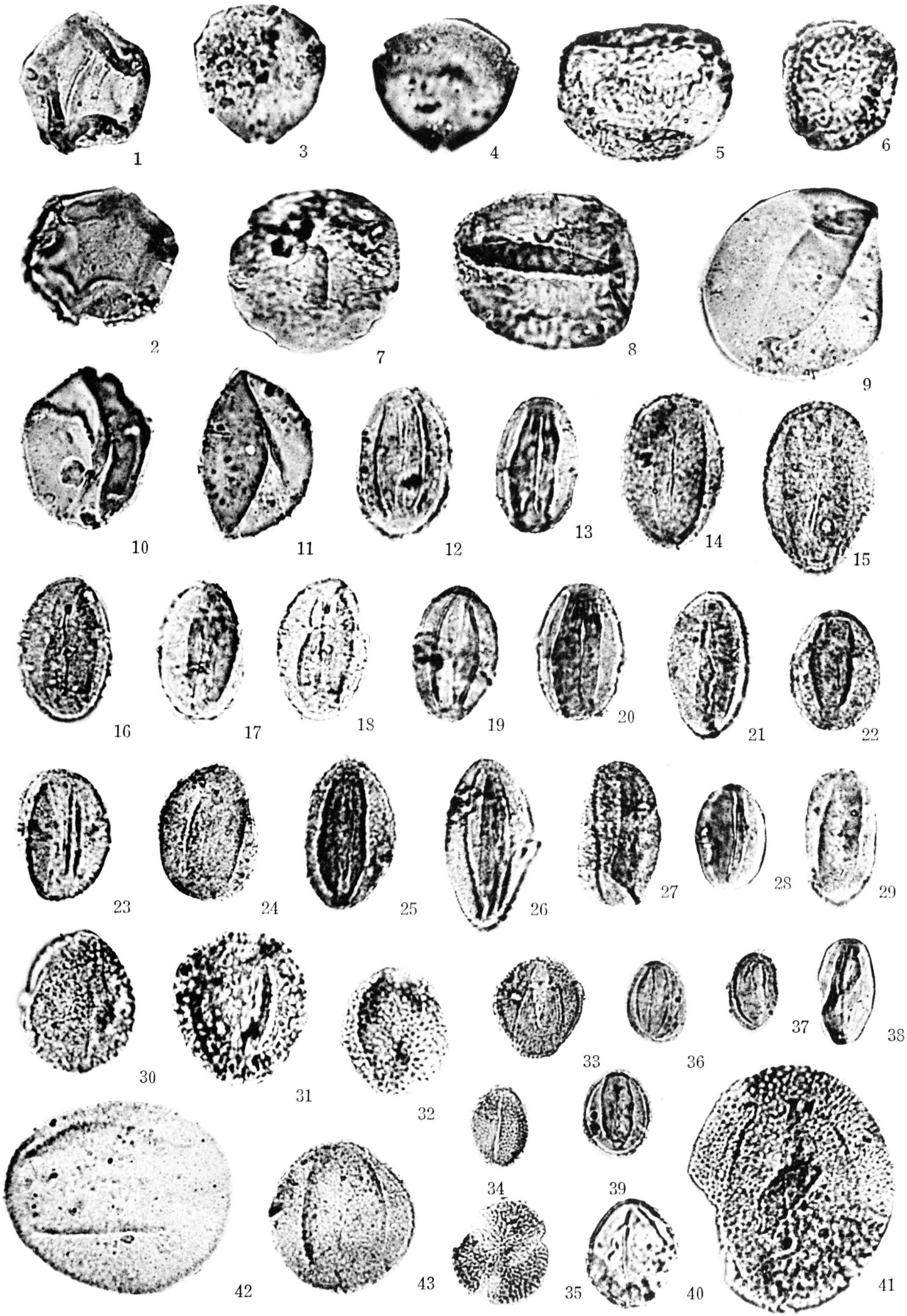

K. Takahashi: Vorläufige Mitteilung über das Problem 


\section{Erklärung zu Tafel 18}

(Alle Objekte ca. $810 \times$ )

Fig. 1-4. Tricolpopoll. meinohamensis meinohamensis $\mathrm{T}_{\mathrm{AK}}$.

Onga-Schichten, Fig. 1, 3, 4: Honishi-Unterflöz, Fig. 2: Nanashaku-Flöz

Fig. 5, 6, 11. Tricolpopoll. meinohamensis rotundus TAK.

Onga-Schichten, Fig. 5: Nanashaku-Flöz, Fig. 6: Yonshaku-Flöz, Fig. 11: Sanjaku-Flöz

Fig. 7-10. Tricolpopoll. chikushiensis grandiformis TAK.

Onga-Schichten, Fig. 7 : Nanashaku-Flöz, Fig. 8: Takae-ezari-Flöz, Fig. 10 : Namae-YohedaFlöz

Fig. 12-15. Tricolpopoll. chikushiensis globulosus TAK.

Onga-Schichten, Fig. 12: Honishi-Unterflöz, Fig. 13; Nanashaku-Flöz, Fig. 14: ShintakaeFlöz, Fig. 15: Oonedo-Yoheda-Flöz

Fig. 16-18, 24, 25. Tricolporopoll. matsushitai TAK.

Onga-Schichten, Fig. 16, 17, 25 : Namae-Yoheda-Flöz, Fig. 18: Sanjaku-Floz, Fig. 24 : Honishi-Unterflöz

Fig. 19. Tricolporopoll. cf. cingulum pusillus (R. PotonIÉ) Ideyama-Schichten

Fig. 20. Tricolporopoll. cingulum oviformis (R. PotoniE) Onga-Schichten, Shintakae-Flöz

Fig. 21. Tricolporopoll. megaexactus exactus (R. PotoniÉ) Onga-Schichten, Shintakae-Flöz

Fig. 22, 23. Tricolporopoll. consularis $\mathrm{T}_{\mathrm{AK}}$.

Onga-Schichten, Fig. 22: cf., Namae-Yoheda-Flöz, Fig. 23: Honishi-Unterflöz

Fig. 26, 27. Tricolporopoll. katsukiensis TAK.

Onga-Schichten, Fig. 26: Shintakae-Flöz, Fig. 27: Sanjaku-Flöz

Fig. 28, 29. Tricolporopoll. ongaensis TAK.

Onga-Schichten, Namae-Yoheda-Flöz

Fig. 30. Tricolporopoll. sp.

Onga-Schichten. Takae-ezari-Flöz

Fig. 31, 32. Tricolporopoll. sp.

Onga-Schichten, Fig. 31: Nanashaku-Flöz, Fig. 32: Honishi-Unterflöz

Fig. 33-37. Tricolporopoll.-Clavoferae-Gruppe

Fig. 35-37: Tricolporopoll. tertiarius TAK.

Ideyama-Schichten, Fig. 33 ; Fig. 35-37: C-B ; Onga-Schichten, Fig. 34: Mie-Unterflöz

Fig. 38-41. Tetradopoll. sp.

Onga-Schichten, Fig. 38: Takae-ezari-Flöz, Fig. 39: Namae-Yoheda-Flöz, Fig. 40: Shintakae-Flöz, Fig. 41: Namae-Flöz 
Mem. Fac. Sci., Kyushu Univ., Ser. D, Vol, IX
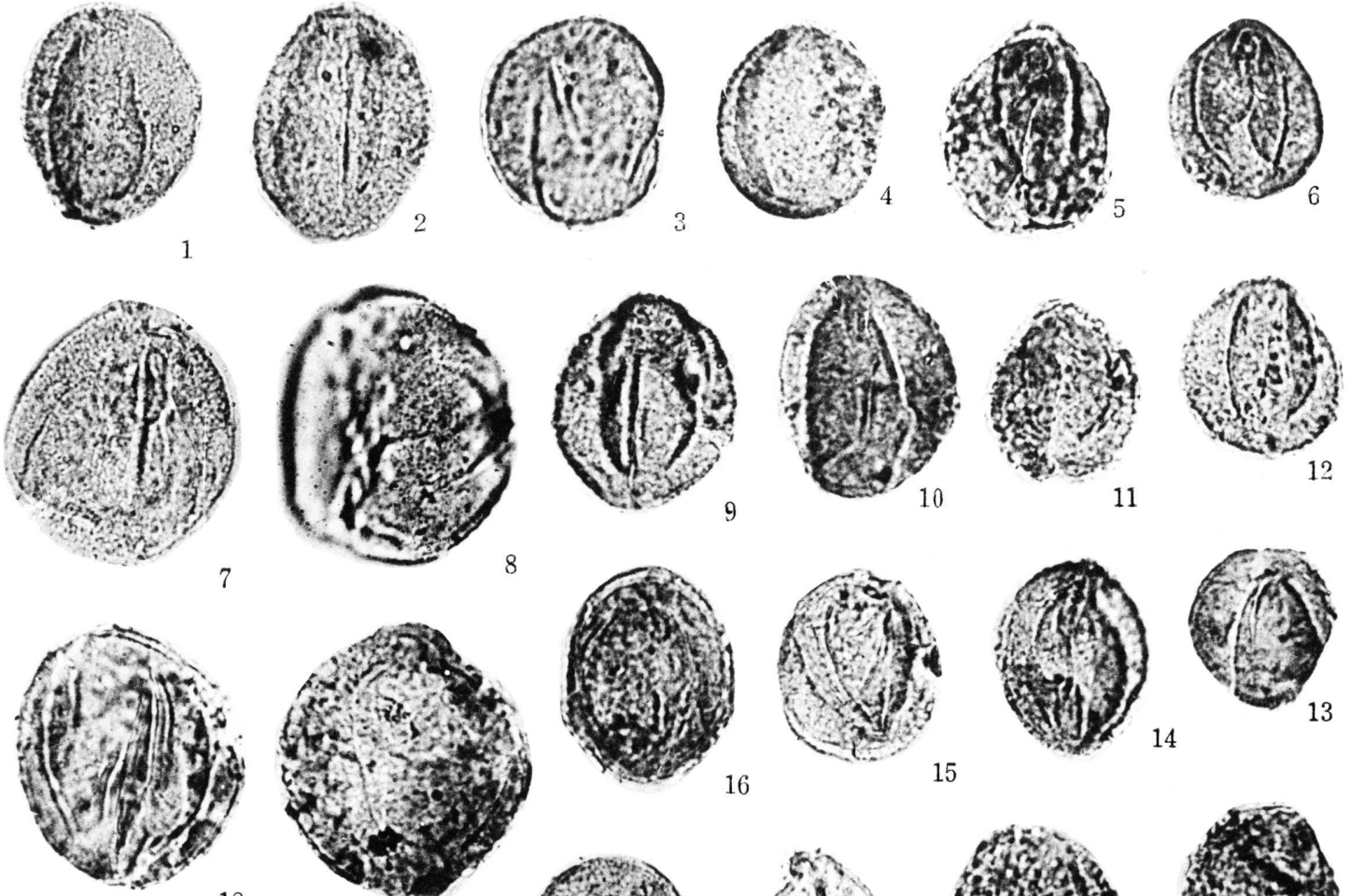

18
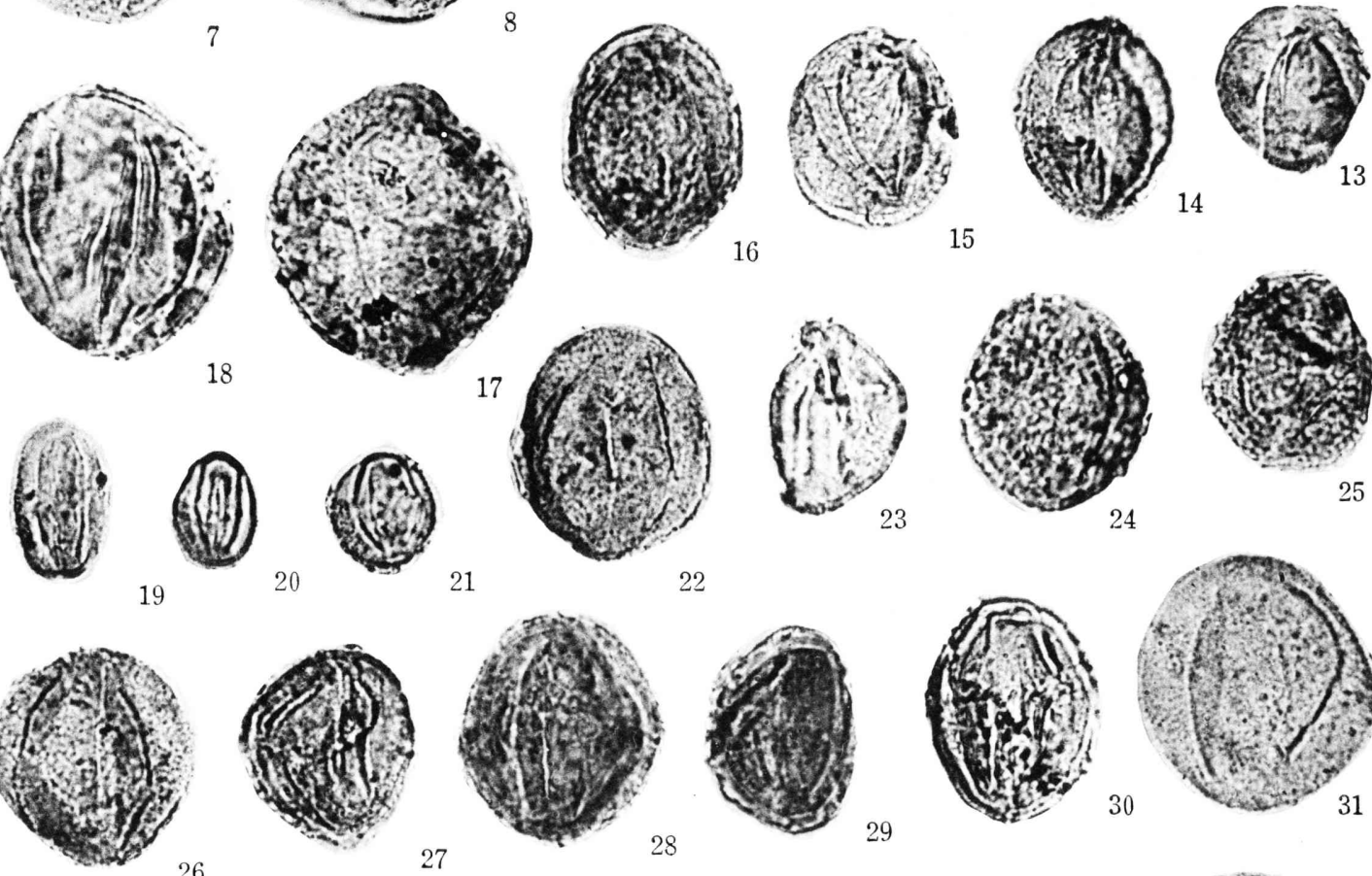

23
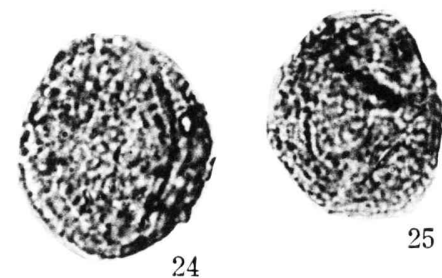

tots

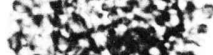

ing

27

28

29
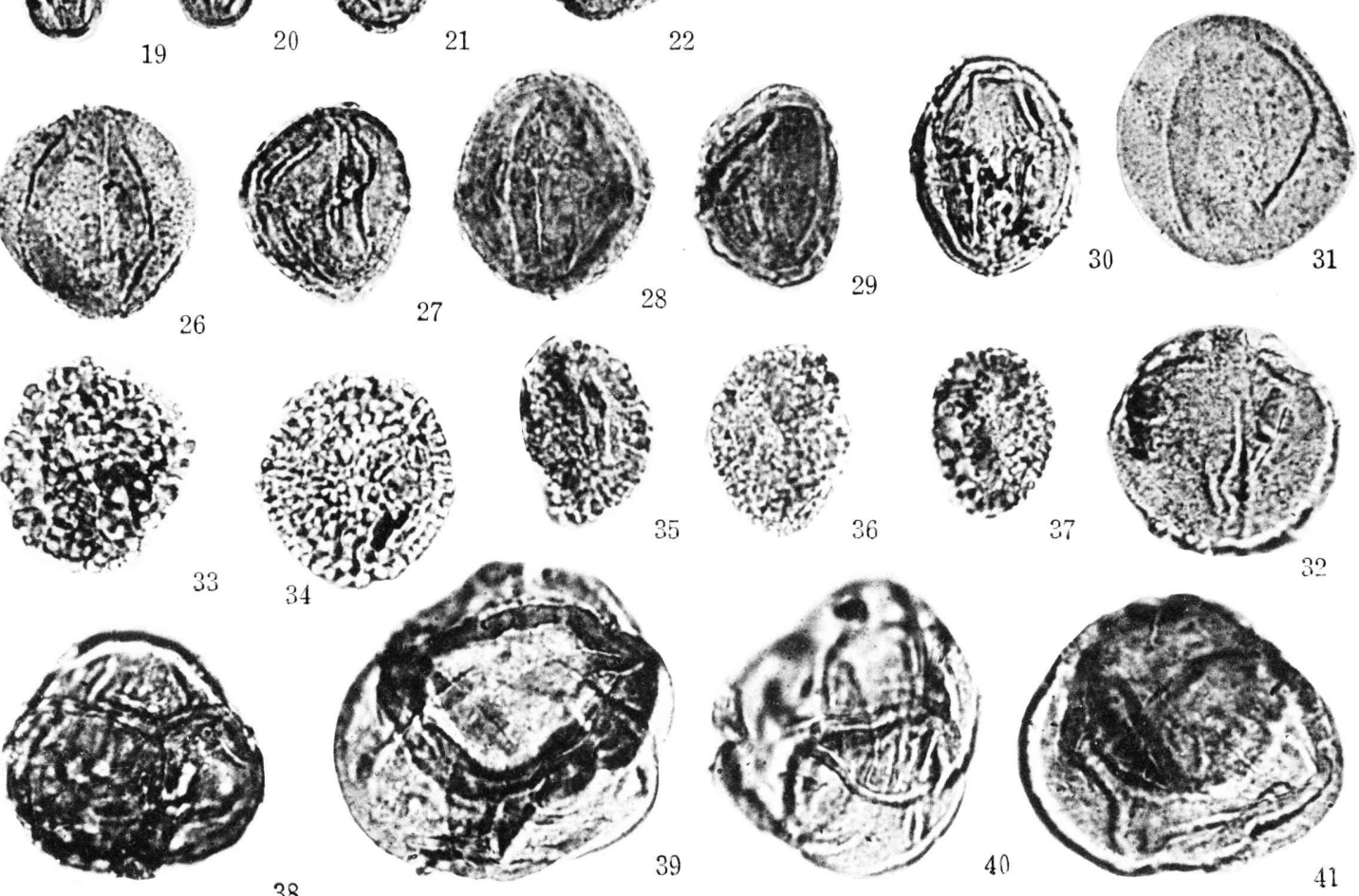

K. Takahashi: Vorläufige Mitteilung über das Problem 